hep-th/0703049

\title{
BRST approach to Lagrangian construction for fermionic higher spin fields in AdS space
}

\author{
I.L. BuchbindeR ${ }^{a *}$ V.A. KRYKhtin ${ }^{a, b \dagger}$, A.A. ReshetnyaK ${ }^{c \ddagger}$ \\ ${ }^{a}$ Department of Theoretical Physics, \\ Tomsk State Pedagogical University, \\ Tomsk 634041, Russia \\ ${ }^{b}$ Laboratory of Mathematical Physics, \\ Tomsk Polytechnic University, \\ Tomsk 634034, Russia \\ ${ }^{c}$ Laboratory of Non-equilibrium State Theory, \\ Institute of Strength Physics and Materials Science, \\ Tomsk 634021, Russia
}

\begin{abstract}
We develop a general gauge-invariant Lagrangian construction for half-integer higher spin fields in the AdS space of any dimension. Starting with a formulation in terms of an auxiliary Fock space, we obtain closed nonlinear symmetry algebras of higher spin fermionic fields in the AdS space and find the corresponding BRST operator. A universal procedure for constructing gauge-invariant Lagrangians describing the dynamics of fermionic fields of any spin is developed. No off-shell constraints for the fields and gauge parameters are imposed from the very beginning. It is shown that all the constraints determining an irreducible representation of the AdS group arise as a consequence of the equations of motion and gauge transformations. As an example of the general procedure, we derive gauge-invariant Lagrangians for massive fermionic fields of spin $1 / 2$ and $3 / 2$ containing the complete set of auxiliary fields and gauge symmetries.
\end{abstract}

\section{Introduction}

Problems of higher spin field theory have attracted much attention for a long time due to the hope of finding new possibilities and approaches to the unification of the fundamental interactions. Higher spin field theory is closely related to superstring theory, which operates with an infinite tower of bosonic and fermionic higher spin fields, including massless and massive

\footnotetext{
*joseph@tspu.edu.ru † ‘krykhtin@mph.phtd.tpu.edu.ru $\quad \ddagger$ reshet@tspu.edu.ru
} 
ones. A Lagrangian formulation for an interacting higher spin field theory is one (perhaps the last) of the unsolved general problems of classical field theory (see, e.g., the reviews [1]).

The current studies in higher spin field theory concern the aspects of Lagrangian formulation in various dimensions, searches for supersymmetric generalizations, finding a correspondence with superstring and $M$ theories and a construction of interacting Lagrangians (see [2]- [9] for massive and [10]- [18] for massless higher spin theories).

It is well-known that a space of constant curvature, in particular, the AdS space, is the simplest non-trivial background providing a consistent propagation of higher spin fields (see, e.g., $[2,19])$. We point out two attractive features of higher spin theory in the AdS space. First, the radius of the AdS space ensures the presence of a natural dimensional parameter for the accommodation of compatible self-interactions for massless higher spin fields [20,21]. Second, higher spin fields in the AdS space are closely related to the conjecture [22] on the AdS/CFT correspondence between the conformal $\mathcal{N}=4 \mathrm{SYM}$ theory and the superstring theory on the $A d S_{5} \times S_{5}$ Ramond-Ramond background [7].

The study of a Lagrangian formulation for massive higher spin field theories was initiated by the pioneering works of Fierz-Pauli and Singh-Hagen [23] for theories in the four-dimensional Minkowski space. These works demonstrated a specific feature of higher spin field theories: all such theories include, apart from the basic fields with a given spin, also some auxiliary fields with lesser spins, which provide a compatibility of the Lagrangian equations of motion with the constraints determining an irreducible representation of the Poincare group. Attempts to construct Lagrangian descriptions of free higher spin field theories in curved spaces and derive higher spin interactions have resulted in consistency problems which generally remain open in spite of numerous efforts.

The present work is devoted to a derivation of general gauge-invariant Lagrangian for massive and massless fields of any half-integer spin in AdS spaces of arbitrary dimensions. Our approach is based on use of the BRST-BFV construction [24] (see also the reviews [25]), which was initially developed for the quantization of gauge theories. Following a tradition accepted in string theory and in higher spin field theory, we further refer to this construction as the BRST construction, and to the corresponding BFV charge, as the BRST charge. The general application of the BRST construction to higher spin field theory consists in three steps. First, the conditions determining the representation with a given spin are regarded as first-class constraints. Second, using the algebra of these constraints, one constructs the BRST charge. Third, a higher spin field Lagrangian is constructed in terms of the BRST charge in such a way that the corresponding equations of motion reproduce the initial constraints. The final step is of fundamental importance and ensures the correctness of the Lagrangian construction. We emphasize that this approach automatically implies a gauge-invariant Lagrangian. One can expect that in the case of massive theories a Lagrangian resulting from the BRST construction should contain a number of Stückelberg fields.

A derivation of Lagrangians for massless bosonic higher spin fields on a basis of the BRST approach has been considered in [26,27] for the flat space and in [28] for the AdS space. A generalization of the BRST approach to Lagrangians of massless fermionic higher spin fields in flat spaces of arbitrary dimensions has been given in [29]. An interaction vertex for massless bosonic higher spin fields in flat and AdS spaces has been studied within the BRST approach in [30]. The first implementation of the BRST construction for the derivation of Lagrangians for massive higher spin fields has been given in our papers for bosonic [31] and fermionic [32] models in Minkowski spaces of arbitrary dimensions and for bosonic models in AdS spaces of arbitrary dimensions [33]. Thus, the BRST approach presents a universal generating method of constructing Lagrangian formulations for higher spin fields.

In this paper, we develop a gauge-invariant approach to a Lagrangian construction for totally 
symmetric fermionic higher spin fields in the AdS space of any dimension 1 . The conditions determining an irreducible representation of the AdS group with a given spin on tensor-spinor fields are reformulated as operator constraints in an auxiliary Fock space. These constraints form a nonlinear superalgebra with a central bosonic charge. Such an algebra has a more complex structure in comparison with its counterpart for bosonic higher spin fields in the AdS space [33], due to the presence of non-vanishing operator structure functions resolving the Jacobi identity, and therefore the construction of the corresponding BRST charge faces a problem. Some aspects of the BRST construction for bosonic nonlinear constraint algebras have been discussed in $[35,36]$. We find a solution of the BRST construction for a nonlinear superalgebra in the theory under consideration and obtain a Lagrangian which reproduces the initial constraints as a consequence of the equations of motion.

The paper is organized as follows. In Section 2, we examine a closed operator superalgebra based on the constraints that determine an irreducible representation of the AdS group with a half-integer spin. In Section 3, we find a superalgebra of the additional parts for the superalgebra of a modified set of the initial operators, obtained by a linear transformation of the initial constraints, and then realize its representation in terms of new (extra) creation and annihilation operators. In Section 4, we calculate a deformed nonlinear superalgebra of constraints enlarged by the additional parts of the modified constraints. A similar construction for fermionic fields in flat space has been given in [29]. Next, we construct a BRST operator corresponding to the superalgebra of the modified enlarged constraints. The derivation of an action and of a sequence of reducible gauge transformations describing the propagation of a fermionic field of an arbitrary spin in the AdS space is realized in Section 5. In Section 6, we prove that the constructed action reproduces the correct conditions for the field that determine an irreducible representation of the AdS group with a fixed half-integer spin. In Section 7, we illustrate the procedure by constructing gauge-invariant Lagrangians for the fields of spin $1 / 2$ and $3 / 2$. In Conclusion, we summarize the results of the work and discuss some open problems.

In addition to the conventions of Refs. [32,33], we use the notation $\varepsilon(A), g h(A)$ for the respective values of Grassmann parity and ghost number of a quantity $A$, and denote by $[A, B\}$ the supercommutator of quantities $A, B$, which in the case of definite values of Grassmann parity is given by $[A, B\}=A B-(-1)^{\varepsilon(A) \varepsilon(B)} B A$.

\section{Auxiliary Fock space for higher spin fields in AdS space-time}

The massive half-integer spin $s=n+\frac{1}{2}$ representations of the AdS group are realized in the space of totally symmetric tensor-spinor fields $\Phi_{\mu_{1} \ldots \mu_{n}}(x)$, the Dirac index being suppressed, satisfying to the conditions (see e.g. [4])

$$
\begin{aligned}
& {\left[i \gamma^{\mu} \nabla_{\mu}-r^{\frac{1}{2}}\left(n+\frac{d}{2}-2\right)-m\right] \Phi_{\mu_{1} \mu_{2} \ldots \mu_{n}}(x)=0,} \\
& \gamma^{\mu} \Phi_{\mu \mu_{2} \ldots \mu_{n}}(x)=0 .
\end{aligned}
$$

where $r=\frac{R}{d(d-1)}$, with $R$ being the scalar curvature of the space-time. We use the metric with the mostly minus signature, and the Dirac's matrices obey the relation

$$
\left\{\gamma_{\mu}, \gamma_{\nu}\right\}=2 g_{\mu \nu}
$$

\footnotetext{
${ }^{1}$ For the sake of completeness, we note that a Lagrangian formulation for massive fields of an arbitrary half-integer spin in the AdS space subject to algebraic constraints has been recently suggested in [34].
} 
In order to avoid an explicit manipulation with the indices, it is convenient to introduce an auxiliary Fock space $2 \mathcal{H}$ generated by creation and annihilation operators with tangent space indices $(a, b=0,1, \ldots, d-1)$

$$
\left[a_{a}, a_{b}^{+}\right]=-\eta_{a b}, \quad \eta_{a b}=\operatorname{diag}(+,-, \ldots,-) .
$$

An arbitrary vector in this Fock space has the form

$$
|\Phi\rangle=\sum_{n=0}^{\infty} \Phi_{a_{1} \ldots a_{n}}(x) a^{+a_{1}} \ldots a^{+a_{n}}|0\rangle=\sum_{n=0}^{\infty} \Phi_{\mu_{1} \ldots \mu_{n}}(x) a^{+\mu_{1}} \ldots a^{+\mu_{n}}|0\rangle,
$$

where $a^{+\mu}(x)=e_{a}^{\mu}(x) a^{+a}, a^{\mu}(x)=e_{a}^{\mu}(x) a^{a} ; e_{a}^{\mu}(x)$ being the vielbein. It is evident that

$$
\left[a_{\mu}, a_{\nu}^{+}\right]=-g_{\mu \nu}
$$

We refer to the vector (5) as the basic vector. The fields $\Phi_{\mu_{1} \ldots \mu_{n}}(x)$ are the coefficient functions of the vector $|\Phi\rangle$ and its symmetry properties are stipulated by the symmetry properties of the product of the creation operators. We also assume the standard relation

$$
\nabla_{\mu} e_{\nu}^{a}=\partial_{\mu} e_{\nu}^{a}-\Gamma_{\mu \nu}^{\lambda} e_{\lambda}^{a}+\omega_{\mu b}^{a} e_{\nu}^{b}=0
$$

We intend to realize relations (11), (2) as certain constraints on the vector $|\Phi\rangle$ (15). To this end, we define an operator $D_{\mu}$ acting on the vectors $|\Phi\rangle$,

$$
D_{\mu}=\partial_{\mu}-\omega_{\mu}^{a b}\left(a_{a}^{+} a_{b}-\frac{1}{4} \gamma_{a b}\right), \quad \partial_{\mu}|0\rangle=0, \quad \gamma_{a b}=\frac{1}{2}\left(\gamma_{a} \gamma_{b}-\gamma_{b} \gamma_{a}\right)
$$

and operators

$$
\begin{aligned}
\tilde{t}_{0} & =i \gamma^{\mu} D_{\mu}-m-r^{\frac{1}{2}}\left(g_{0}-2\right) \\
\tilde{t}_{1} & =\gamma^{\mu} a_{\mu} \\
g_{0} & =-a^{+\mu} a_{\mu}+\frac{d}{2}
\end{aligned}
$$

We can see that the constraints

$$
\tilde{t}_{0}|\Phi\rangle=\tilde{t}_{1}|\Phi\rangle=0
$$

for the basic vector (5) are equivalent to equations (11), (2), with each component $\Phi_{\mu_{1} \ldots \mu_{n}}(x)$ in (5) subject to (11), (2), and therefore they describe a field of spin $n+1 / 2$.

Because of the fermionic nature of equations (11), (2) with respect to the standard Grassmann parity, and due to the bosonic nature of the operators $\tilde{t}_{0}, \tilde{t}_{1}: \varepsilon\left(\tilde{t}_{0}\right)=\varepsilon\left(\tilde{t}_{1}\right)=0$, in order to equivalently transform these operators into fermionic ones, we now introduce a set of $d+1$ Grassmann-odd "gamma-matrix-like objects" $\tilde{\gamma}^{\mu}, \tilde{\gamma}$ subject to 3

$$
\left\{\tilde{\gamma}^{\mu}, \tilde{\gamma}^{\nu}\right\}=2 g^{\mu \nu}, \quad\left\{\tilde{\gamma}^{\mu}, \tilde{\gamma}\right\}=0, \quad \tilde{\gamma}^{2}=-1
$$

and related to the conventional gamma-matrices as follows:

$$
\gamma^{\mu}=\tilde{\gamma}^{\mu} \tilde{\gamma}
$$

\footnotetext{
${ }^{2}$ For more details, see [33].

${ }^{3}$ For more details, see [32].
} 
In terms of these gamma-matrices, we define the Grassmann-odd constraints

$$
\tilde{t}_{0}^{\prime}=-i \tilde{\gamma}^{\mu} D_{\mu}+\tilde{\gamma}\left(m+r^{\frac{1}{2}}\left(g_{0}-2\right)\right), \quad t_{1}=\tilde{\gamma}^{\mu} a_{\mu},
$$

related to (91), (10) as follows:

$$
\tilde{t}_{0}^{\prime}=-\tilde{\gamma} \tilde{t}_{0}, \quad t_{1}=\tilde{\gamma} \tilde{t}_{1}
$$

In order to obtain a Hermitian BRST operator, being the central object of the Lagrangian construction in the BRST approach, we need a set of first-class constraints which must be invariant with respect to Hermitian conjugation and must form a superalgebra with respect to supercommutator multiplication. To this end, we define an odd scalar product:

$$
\langle\tilde{\Psi} \mid \Phi\rangle=\int d^{d} x \sqrt{|g|} \sum_{n, k=0}^{\infty}\left\langle 0\left|a^{\nu_{1}} \ldots a^{\nu_{k}} \Psi_{\nu_{1} \ldots \nu_{k}}^{+}(x) \tilde{\gamma}_{0} \Phi_{\mu_{1} \ldots \mu_{n}}(x) a^{+\mu_{1}} \ldots a^{+\mu_{n}}\right| 0\right\rangle
$$

The operators $\tilde{t}_{0}^{\prime}, t_{1}(15)$ and $t_{1}^{+}=\tilde{\gamma}^{\mu} a_{\mu}^{+}$, being the Hermitian conjugate of $t_{1}$ with respect to the scalar product (17), generate an operator superalgebra with the central charge $\tilde{m}=\left(m-2 r^{\frac{1}{2}}\right)$ and the following set of operators:

$$
\begin{array}{cc}
\tilde{t}_{0}^{\prime}=-i \tilde{\gamma}^{\mu} D_{\mu}+\tilde{\gamma}\left(m+r^{\frac{1}{2}}\left(g_{0}-2\right)\right), & \\
t_{1}=\tilde{\gamma}^{\mu} a_{\mu}, & t_{1}^{+}=\tilde{\gamma}^{\mu} a_{\mu}^{+}, \\
l_{1}=-i a^{\mu} D_{\mu}, & l_{1}^{+}=-i a^{+\mu} D_{\mu}, \\
l_{2}=\frac{1}{2} a^{\mu} a_{\mu}, & l_{2}^{+}=\frac{1}{2} a^{+\mu} a_{\mu}^{+}, \\
& g_{0}=-a_{\mu}^{+} a^{\mu}+\frac{d}{2}, \\
\tilde{l}_{0}^{\prime}=g^{\mu \nu}\left(D_{\nu} D_{\mu}-\Gamma_{\mu \nu}^{\sigma} D_{\sigma}\right)-r\left(g_{0}+t_{1}^{+} t_{1}+\frac{d(d-3)}{4}\right)+\left(m+r^{\frac{1}{2}}\left(g_{0}-2\right)\right)^{2},
\end{array}
$$

which is invariant under Hermitian conjugation. The operators (18)-(23) form an algebra given by Table 1, where

$$
\begin{aligned}
{\left[\tilde{t}_{0}^{\prime}, t_{1}^{+}\right\} } & =2 l_{1}^{+}+\tilde{\gamma} r^{\frac{1}{2}} t_{1}^{+} \\
{\left[\tilde{t}_{0}^{\prime}, t_{1}\right\} } & =2 l_{1}-\tilde{\gamma} r^{\frac{1}{2}} t_{1} \\
{\left[t_{1}^{+}, l_{1}\right\} } & =\left[l_{1}^{+}, t_{1}\right\}=\tilde{t}_{0}^{\prime}-\tilde{\gamma}\left(\tilde{m}+r^{\frac{1}{2}} g_{0}\right) \\
{\left[\tilde{t}_{0}^{\prime}, l_{1}\right\} } & =r\left(2 t_{1}^{+} l_{2}+g_{0} t_{1}-\frac{1}{2} t_{1}\right)-\tilde{\gamma} r^{\frac{1}{2}} l_{1}, \\
{\left[l_{1}^{+}, \tilde{t}_{0}^{\prime}\right\} } & =r\left(2 l_{2}^{+} t_{1}+t_{1}^{+} g_{0}-\frac{1}{2} t_{1}^{+}\right)-\tilde{\gamma} r^{\frac{1}{2}} l_{1}^{+}, \\
{\left[t_{1}, \tilde{l}_{0}^{\prime}\right\} } & =r\left(4 t_{1}^{+} l_{2}+2 g_{0} t_{1}-t_{1}\right)-r^{\frac{1}{2}} \tilde{m} t_{1}, \\
{\left[\tilde{l}_{0}^{\prime}, t_{1}^{+}\right\} } & =4 r\left(l_{2}^{+} t_{1}+t_{1}^{+} g_{0}\right)+2 r^{\frac{1}{2}} \tilde{m} t_{1}^{+}, \\
{\left[l_{1}, \tilde{l}_{0}^{\prime}\right\} } & =4 r\left(l_{1}^{+} l_{2}+g_{0} l_{1}\right)+2 r^{\frac{1}{2}} \tilde{m} l_{1}, \\
{\left[\tilde{l}_{0}^{\prime}, l_{1}^{+}\right\} } & =4 r\left(l_{2}^{+} l_{1}+l_{1}^{+} g_{0}\right)+2 r^{\frac{1}{2}} \tilde{m} l_{1}^{+}, \\
{\left[l_{2}, \tilde{l}_{0}^{\prime}\right\} } & =4 r^{\frac{1}{2}}\left(r^{\frac{1}{2}}\left(1+g_{0}\right)+\tilde{m}\right) l_{2}, \\
{\left[\tilde{l}_{0}^{\prime}, l_{2}^{+}\right\} } & =4 r^{\frac{1}{2}} l_{2}^{+}\left(r^{\frac{1}{2}}\left(1+g_{0}\right)+\tilde{m}\right) \\
{\left[l_{1}, l_{1}^{+}\right\} } & =\tilde{l}_{0}^{\prime}-\tilde{m}^{2}-2 r^{\frac{1}{2}} \tilde{m} g_{0}+r\left(\frac{3}{2} t_{1}^{+} t_{1}-\frac{1}{2} g_{0}-4 l_{2}^{+} l_{2}\right) .
\end{aligned}
$$

We call this algebra the massive half-integer higher spin symmetry algebra in the AdS space. 


\begin{tabular}{|c|c|c|c|c|c|c|c|c|c|c|}
\hline$[\downarrow, \rightarrow\}$ & $\tilde{t}_{0}^{\prime}$ & $t_{1}$ & $t_{1}^{+}$ & $\tilde{l}_{0}^{\prime}$ & $l_{1}$ & $l_{1}^{+}$ & $l_{2}$ & $l_{2}^{+}$ & $g_{0}$ & $\tilde{m}$ \\
\hline$\tilde{t}_{0}^{\prime}$ & $-2 \tilde{l}_{0}^{\prime}$ & (25) & (24) & 0 & (27) & $-(28)$ & $-2 \tilde{\gamma} r^{\frac{1}{2}} l_{2}$ & $2 \tilde{\gamma} r^{\frac{1}{2}} l_{2}^{+}$ & 0 & 0 \\
\hline$t_{1}$ & (25) & $4 l_{2}$ & $-2 g_{0}$ & (29) & 0 & $-(\underline{26})$ & 0 & $-t_{1}^{+}$ & $t_{1}$ & 0 \\
\hline$t_{1}^{+}$ & (24) & $-2 g_{0}$ & $4 l_{2}^{+}$ & $-(30)$ & (26) & 0 & $t_{1}$ & 0 & $-t_{1}^{+}$ & 0 \\
\hline$\tilde{l}_{0}^{\prime}$ & 0 & $-(29)$ & (30) & 0 & $-(31)$ & (32) & $-(33)$ & (34) & 0 & 0 \\
\hline$l_{1}$ & $-(27)$ & 0 & $-(26)$ & (31) & 0 & (35) & 0 & $-l_{1}^{+}$ & $l_{1}$ & 0 \\
\hline$l_{1}^{+}$ & (28) & (26) & 0 & $-(32)$ & $-(35)$ & 0 & $l_{1}$ & 0 & $-l_{1}^{+}$ & 0 \\
\hline$l_{2}$ & $2 \tilde{\gamma} r^{\frac{1}{2}} l_{2}$ & 0 & $-t_{1}$ & (33) & 0 & $-l_{1}$ & 0 & $g_{0}$ & $2 l_{2}$ & 0 \\
\hline$l_{2}^{+}$ & $-2 \tilde{\gamma} r^{\frac{1}{2}} l_{2}^{+}$ & $t_{1}^{+}$ & 0 & $-(34)$ & $l_{1}^{+}$ & 0 & $-g_{0}$ & 0 & $-2 l_{2}^{+}$ & 0 \\
\hline$g_{0}$ & 0 & $-t_{1}$ & $t_{1}^{+}$ & 0 & $-l_{1}$ & $l_{1}^{+}$ & $-2 l_{2}$ & $2 l_{2}^{+}$ & 0 & 0 \\
\hline$\tilde{m}$ & 0 & 0 & 0 & 0 & 0 & 0 & 0 & 0 & 0 & 0 \\
\hline
\end{tabular}

Table 1: The algebra of the initial operators.

The method of Lagrangian construction requires an enlarging of the initial constraints $o_{i}$ $\left(\tilde{t}_{0}^{\prime}, t_{1} \in\left\{o_{i}\right\}\right)$, so that the enlarged Hermitian operators contain arbitrary parameters and the set of enlarged operators form an (super)algebra. A procedure of constructing of these enlarged constraints $O_{i}=o_{i}+o_{i}^{\prime}$ for the operators $o_{i}$ is considerably simplified if the initial operators $o_{i}$ (super)commute with its additional parts $o_{i}^{\prime}:\left[o_{i}, o_{j}^{\prime}\right\}=0$, defined in the auxiliary operator space. In this case we can apply the method elaborated in [33]. The such realization of the enlarged first-class constraints $O_{i}$, by means of an additive composition of the quantities $o_{i}$ with additional parts $o_{i}^{\prime}$, requires a transformation of $o_{i}$ into an equivalent set of constraints $\tilde{o}_{i}$ which do not contain the $\tilde{\gamma}$-matrix, because the set $o_{i}^{\prime}$ will contain this object by construction. Thus, in order to simplify the subsequent calculations, we choose the operators $\tilde{o}_{i}$ related by a nondegenerate linear transformation $o_{i}=U_{j}^{i} \tilde{o}_{j}$ with the initial constraints reproducing relations (11), (21),

$$
\begin{aligned}
& t_{0}=-i \tilde{\gamma}^{\mu} D_{\mu} \\
& l_{0}=g^{\mu \nu}\left(D_{\nu} D_{\mu}-\Gamma_{\mu \nu}^{\sigma} D_{\sigma}\right)-r\left(g_{0}+t_{1}^{+} t_{1}+\frac{d(d-3)}{4}\right) .
\end{aligned}
$$

The other constraints coincide with the initial ones.

\footnotetext{
${ }^{4}$ Note that the case of a two-parametric set of constraints determined by the operator $\tilde{t}_{0 \alpha}^{\prime}=-i \tilde{\gamma}^{\mu} D_{\mu}+$ $\alpha_{1} \tilde{\gamma} \tilde{m}+\alpha_{2} \tilde{\gamma} r^{\frac{1}{2}} g_{0}$, analogous to the consideration for bosonic fields in the AdS space [33], from which it would be possible to construct a Lagrangian that reproduces Eq. (12) for any values $\alpha_{1}, \alpha_{2}$, can be realized by means of the above nondegenerate transformation of the superalgebra of the initial constraints.
} 


\begin{tabular}{||c||c|c|c|c|c|c|c|c||c|c||}
\hline \hline$[\downarrow, \rightarrow\}$ & $t_{0}$ & $t_{1}$ & $t_{1}^{+}$ & $l_{0}$ & $l_{1}$ & $l_{1}^{+}$ & $l_{2}$ & $l_{2}^{+}$ & $g_{0}$ & $\tilde{m}$ \\
\hline \hline$t_{0}$ & $-2 l_{0}$ & $2 l_{1}$ & $2 l_{1}^{+}$ & 0 & $(38)$ & $-(41)$ & 0 & 0 & 0 & 0 \\
\hline$t_{1}$ & $2 l_{1}$ & $4 l_{2}$ & $-2 g_{0}$ & $(\sqrt{39})$ & 0 & $-t_{0}$ & 0 & $-t_{1}^{+}$ & $t_{1}$ & 0 \\
\hline$t_{1}^{+}$ & $2 l_{1}^{+}$ & $-2 g_{0}$ & $4 l_{2}^{+}$ & $-(44)$ & $t_{0}$ & 0 & $t_{1}$ & 0 & $-t_{1}^{+}$ & 0 \\
\hline$l_{0}$ & 0 & $-(39)$ & $(42)$ & 0 & $-(40)$ & $(43)$ & 0 & 0 & 0 & 0 \\
\hline$l_{1}$ & $-(38)$ & 0 & $-t_{0}$ & $(40)$ & 0 & $(44)$ & 0 & $-l_{1}^{+}$ & $l_{1}$ & 0 \\
\hline$l_{1}^{+}$ & $(41)$ & $t_{0}$ & 0 & $-(43)$ & $-(44)$ & 0 & $l_{1}$ & 0 & $-l_{1}^{+}$ & 0 \\
\hline$l_{2}$ & 0 & 0 & $-t_{1}$ & 0 & 0 & $-l_{1}$ & 0 & $g_{0}$ & $2 l_{2}$ & 0 \\
\hline$l_{2}^{+}$ & 0 & $t_{1}^{+}$ & 0 & 0 & $l_{1}^{+}$ & 0 & $-g_{0}$ & 0 & $-2 l_{2}^{+}$ & 0 \\
\hline \hline$g_{0}$ & 0 & $-t_{1}$ & $t_{1}^{+}$ & 0 & $-l_{1}$ & $l_{1}^{+}$ & $-2 l_{2}$ & $2 l_{2}^{+}$ & 0 & 0 \\
\hline$\tilde{m}$ & 0 & 0 & 0 & 0 & 0 & 0 & 0 & 0 & 0 & 0 \\
\hline \hline
\end{tabular}

Table 2: The algebra of the modified initial operators.

The operators $\tilde{o}_{i}$ given by (19) $-(22),(\underline{36}),(37)$ with the central charge $\tilde{m}$ form a superalgebra given by Table 2, where

$$
\begin{aligned}
{\left[t_{0}, l_{1}\right\} } & =r\left(2 t_{1}^{+} l_{2}+g_{0} t_{1}-\frac{1}{2} t_{1}\right), \\
{\left[t_{1}, l_{0}\right\} } & =r\left(4 t_{1}^{+} l_{2}+2 g_{0} t_{1}-t_{1}\right), \\
{\left[l_{1}, l_{0}\right\} } & =r\left(4 l_{1}^{+} l_{2}+2 g_{0} l_{1}-l_{1}\right), \\
{\left[l_{1}^{+}, t_{0}\right\} } & =r\left(2 l_{2}^{+} t_{1}+t_{1}^{+} g_{0}-\frac{1}{2} t_{1}^{+}\right), \\
{\left[l_{0}, t_{1}^{+}\right\} } & =r\left(4 l_{2}^{+} t_{1}+2 t_{1}^{+} g_{0}-t_{1}^{+}\right), \\
{\left[l_{0}, l_{1}^{+}\right\} } & =r\left(4 l_{2}^{+} l_{1}+2 l_{1}^{+} g_{0}-l_{1}^{+}\right), \\
{\left[l_{1}, l_{1}^{+}\right\} } & =l_{0}+r\left(g_{0}^{2}-\frac{1}{2} g_{0}-4 l_{2}^{+} l_{2}+\frac{3}{2} t_{1}^{+} t_{1}\right) .
\end{aligned}
$$

In terms of the operators $\tilde{o}_{i}$, equations (12) have the form

$$
\left[t_{0}+\tilde{\gamma} m+\tilde{\gamma} r^{\frac{1}{2}}\left(g_{0}-2\right)\right]|\Phi\rangle=0, \quad t_{1}|\Phi\rangle=0
$$

In what follows, we shall demonstrate the construction of Lagrangians by using the BRST approach developed for bosonic fields in the AdS space in [33] so as to reproduce equations (12), or, equivalently, (45). According to our approach, we have to extend the operators $\tilde{o}_{i}$ of the algebra given by Table 2 by additional parts $o_{i}^{\prime}: \tilde{o}_{i} \rightarrow \tilde{O}_{i}=\tilde{o}_{i}+o_{i}^{\prime}$, so that 1) $\tilde{O}_{i}$ must be in involution $\left[\tilde{O}_{i}, \tilde{O}_{j}\right\} \sim \tilde{O}_{k}$ and 2) each Hermitian operator must contain linearly an arbitrary parameter whose values are to be determined later (for details, see $[29,32,33]$ ).

The next step of this procedure is to find the additional parts $o_{i}^{\prime}$ for the operators (19)-(22), (36), (37). 


\section{Additional parts of operators}

As stated at the end of the previous section, now we intend to find the additional parts $o_{i}^{\prime}$ for the operators (19)-(22), (36), (37). Following the procedure described in the bosonic case in [33], we must first find the superalgebra of the additional parts. Then these additional parts are constructed from new (additional) creation and annihilation operators, as well as from the constants of the theory $r, m$, and from one of the gamma-matrix-like objects $\tilde{\gamma}$. Furthermore, we must introduce linearly 5 into $t_{0}^{\prime}, g_{0}^{\prime}$ (these are the additional parts for $t_{0}, g_{0}$, respectively) some arbitrary constants whose values will be determined later by the condition that equations (45) be reproduced.

Let us briefly remind and at the same time generalize the method given in [33] to algebras including fermionic operators. The (super)commutation relations for the operators $\tilde{o}_{i}$ from Table 2 have the structure

$$
\left[\tilde{o}_{i}, \tilde{o}_{j}\right\}_{s}=f_{i j}^{k} \tilde{o}_{k}+f_{i j}^{k m} \tilde{o}_{k} \tilde{O}_{m}
$$

where the constants $f_{i j}^{k}, f_{i j}^{k m}$ obey the properties $\left(f_{i j}^{k}, f_{i j}^{k m}\right)=-(-1)^{\varepsilon\left(O_{i}\right) \varepsilon\left(O_{j}\right)}\left(f_{j i}^{k}, f_{j i}^{k m}\right)$. Then we suppose that the operators $\tilde{o}_{i}$ supercommute with the additional parts $o_{i}^{\prime}$. In this case, one can check that if we define the superalgebra of the additional parts in the form

$$
\left[o_{i}^{\prime}, o_{j}^{\prime}\right\}_{s}=f_{i j}^{k} o_{k}^{\prime}-(-1)^{\varepsilon\left(\tilde{o}_{k}\right) \varepsilon\left(\tilde{o}_{m}\right)} f_{i j}^{k m} o_{m}^{\prime} o_{k}^{\prime}
$$

then the enlarged operators $\tilde{O}_{i}=\tilde{o}_{i}+o_{i}^{\prime}$ will form a closed superalgebra

$$
\left[\tilde{O}_{i}, \tilde{O}_{j}\right\}_{s}=f_{i j}^{k} \tilde{O}_{k}-\left(f_{i j}^{m k}+(-1)^{\varepsilon\left(O_{k}\right) \varepsilon\left(O_{m}\right)} f_{i j}^{k m}\right) o_{m}^{\prime} \tilde{O}_{k}+f_{i j}^{k m} \tilde{O}_{k} \tilde{O}_{m}
$$

which is deformed in comparison with (46)).

After applying of the procedure above, the superalgebra of the additional parts takes the form given by Table 3, where

$$
\begin{aligned}
& {\left[l_{1}^{\prime}, t_{0}^{\prime}\right\}=r\left(2 t_{1}^{\prime+} l_{2}^{\prime}+g_{0}^{\prime} t_{1}^{\prime}-\frac{1}{2} t_{1}^{\prime}\right),} \\
& {\left[t_{0}^{\prime}, l_{1}^{\prime+}\right\}=r\left(2 l_{2}^{\prime+} t_{1}^{\prime}+t_{1}^{\prime+} g_{0}^{\prime}-\frac{1}{2} t_{1}^{\prime+}\right),} \\
& {\left[l_{0}^{\prime}, t_{1}^{\prime}\right\}=r\left(4 t_{1}^{\prime+} l_{2}^{\prime}+2 g_{0}^{\prime} t_{1}^{\prime}-t_{1}^{\prime}\right),} \\
& {\left[t_{1}^{\prime+}, l_{0}^{\prime}\right\}=r\left(4 l_{2}^{\prime+} t_{1}^{\prime}+2 t_{1}^{\prime+} g_{0}^{\prime}-t_{1}^{\prime+}\right),} \\
& {\left[l_{0}^{\prime}, l_{1}^{\prime}\right\}=r\left(4 l_{1}^{\prime+} l_{2}^{\prime}+2 g_{0}^{\prime} l_{1}^{\prime}-l_{1}^{\prime}\right),} \\
& {\left[l_{1}^{\prime+}, l_{0}^{\prime}\right\}=r\left(4 l_{2}^{\prime+} l_{1}^{\prime}+2 l_{1}^{\prime+} g_{0}^{\prime}-l_{1}^{\prime+}\right),} \\
& {\left[l_{1}^{\prime}, l_{1}^{++}\right\}=l_{0}^{\prime}-r\left(g_{0}^{\prime 2}-\frac{1}{2} g_{0}^{\prime}-4 l_{2}^{\prime+} l_{2}^{\prime}+\frac{3}{2} t_{1}^{\prime+} t_{1}^{\prime}\right) .}
\end{aligned}
$$

In accordance with our method, we ascribe the following value to the additional central charge: $\tilde{m}^{\prime}=-\tilde{m}$, so that the enlarged central charge $\tilde{M}$ becomes equal to zero.

Explicit expressions for the additional parts can be found by the method described in the papers $[29,36]$ and extended to the case of the Verma module construction for a nonlinear superalgebra given by Table 3. Omitting tedious calculations, we have

$$
\begin{array}{ll}
t_{1}^{\prime+}=f^{+}+2 b_{2}^{+} f, & l_{1}^{\prime+}=m_{1} b_{1}^{+}, \\
g_{0}^{\prime}=b_{1}^{+} b_{1}+2 b_{2}^{+} b_{2}+f^{+} f+h, & l_{2}^{\prime+}=b_{2}^{+},
\end{array}
$$

\footnotetext{
${ }^{5}$ We have to introduce linearly some arbitrary constants into all of the additional parts corresponding to Hermitian operators. Since $t_{0}^{2}=-l_{0}$, we cannot obtain an independent arbitrary constant in $l_{0}^{\prime}$.
} 


\begin{tabular}{||c||r|r|r|r|r|r|r|r||r|}
\hline \hline$[\downarrow, \rightarrow\}$ & $t_{0}^{\prime}$ & $t_{1}^{\prime}$ & $t_{1}^{\prime+}$ & $l_{0}^{\prime}$ & $l_{1}^{\prime}$ & $l_{1}^{\prime+}$ & $l_{2}^{\prime}$ & $l_{2}^{\prime+}$ & $g_{0}^{\prime}$ \\
\hline \hline$t_{0}^{\prime}$ & $-2 l_{0}^{\prime}$ & $2 l_{1}^{\prime}$ & $2 l_{1}^{\prime+}$ & 0 & $-(\underline{49})$ & $(\underline{50})$ & 0 & 0 & 0 \\
\hline$t_{1}^{\prime}$ & $2 l_{1}^{\prime}$ & $4 l_{2}^{\prime}$ & $-2 g_{0}^{\prime}$ & $-(\underline{51})$ & 0 & $-t_{0}^{\prime}$ & 0 & $-t_{1}^{\prime+}$ & $t_{1}^{\prime}$ \\
\hline$t_{1}^{\prime+}$ & $2 l_{1}^{\prime+}$ & $-2 g_{0}^{\prime}$ & $4 l_{2}^{\prime+}$ & $(\underline{52})$ & $t_{0}^{\prime}$ & 0 & $t_{1}^{\prime}$ & 0 & $-t_{1}^{\prime+}$ \\
\hline$l_{0}^{\prime}$ & 0 & $(\underline{51})$ & $-(\underline{52})$ & 0 & $(\underline{53})$ & $-(\underline{54})$ & 0 & 0 & 0 \\
\hline$l_{1}^{\prime}$ & $(49)$ & 0 & $-t_{0}^{\prime}$ & $-(\underline{53})$ & 0 & $(\underline{55})$ & 0 & $-l_{1}^{\prime+}$ & $l_{1}^{\prime}$ \\
\hline$l_{1}^{\prime+}$ & $-(50)$ & $t_{0}^{\prime}$ & 0 & $(\underline{54})$ & $-(\underline{55})$ & 0 & $l_{1}^{\prime}$ & 0 & $-l_{1}^{\prime+}$ \\
\hline$l_{2}^{\prime}$ & 0 & 0 & $-t_{1}^{\prime}$ & 0 & 0 & $-l_{1}^{\prime}$ & 0 & $g_{0}^{\prime}$ & $2 l_{2}^{\prime}$ \\
\hline$l_{2}^{\prime+}$ & 0 & $t_{1}^{\prime+}$ & 0 & 0 & $l_{1}^{\prime+}$ & 0 & $-g_{0}^{\prime}$ & 0 & $-2 l_{2}^{\prime+}$ \\
\hline \hline$g_{0}^{\prime}$ & 0 & $-t_{1}^{\prime}$ & $t_{1}^{\prime+}$ & 0 & $-l_{1}^{\prime}$ & $l_{1}^{\prime+}$ & $-2 l_{2}^{\prime}$ & $2 l_{2}^{\prime+}$ & 0 \\
\hline \hline
\end{tabular}

Table 3: The algebra of the additional parts for the operators.

$$
\begin{aligned}
t_{0}^{\prime}=2 m_{1} b_{1}^{+} f-\frac{m_{1}}{2}\left(f^{+}-2 b_{2}^{+} f\right) b_{1}^{+} \sum_{k=1}^{\infty}\left(\frac{-2 r}{m_{1}^{2}}\right)^{k} \frac{\left(b_{2}^{+}\right)^{k-1} b_{1}^{2 k}}{(2 k) !}+\tilde{\gamma} m_{0} \sum_{k=0}^{\infty}\left(\frac{-2 r}{m_{1}^{2}}\right)^{k} \frac{\left(b_{2}^{+}\right)^{k} b_{1}^{2 k}}{(2 k) !} \\
+\frac{r\left(h-\frac{1}{2}\right)}{m_{1}}\left(f^{+}-2 b_{2}^{+} f\right) \sum_{k=0}^{\infty}\left(\frac{-2 r}{m_{1}^{2}}\right)^{k} \frac{\left(b_{2}^{+}\right)^{k} b_{1}^{2 k+1}}{(2 k+1) !} \\
t_{1}^{\prime}=-2 g_{0}^{\prime} f-\left(f^{+}-2 b_{2}^{+} f\right) b_{2}+\frac{1}{2}\left(h-\frac{1}{2}\right)\left(f^{+}-2 b_{2}^{+} f\right) \sum_{k=1}^{\infty}\left(\frac{-2 r}{m_{1}^{2}}\right)^{k} \frac{\left(b_{2}^{+}\right)^{k-1} b_{1}^{2 k}}{(2 k) !} \\
\quad+\frac{1}{2}\left(f^{+}-2 b_{2}^{+} f\right) b_{1}^{+} \sum_{k=1}^{\infty}\left(\frac{-2 r}{m_{1}^{2}}\right)^{k} \frac{\left(b_{2}^{+}\right)^{k-1} b_{1}^{2 k+1}}{(2 k+1) !} \\
\quad-\frac{\tilde{\gamma} m_{0}}{m_{1}} \sum_{k=0}^{\infty}\left(\frac{-2 r}{m_{1}^{2}}\right)^{k} \frac{\left(b_{2}^{+}\right)^{k} b_{1}^{2 k+1}}{(2 k+1) !}
\end{aligned}
$$$$
l_{0}^{\prime}=m_{0}^{2}-r \frac{\tilde{\gamma} m_{0}}{m_{1}}\left(f^{+}-2 b_{2}^{+} f\right) \sum_{k=1}^{\infty}\left(\frac{-8 r}{m_{1}^{2}}\right)^{k} \frac{\left(b_{2}^{+}\right)^{k} b_{1}^{2 k+1}}{(2 k+1) !}\left(1-4^{-k}\right)
$$$$
-r b_{1}^{+} \sum_{k=0}^{\infty}\left(\frac{-8 r}{m_{1}^{2}}\right)^{k} \frac{\left(b_{2}^{+}\right)^{k} b_{1}^{2 k+1}}{(2 k+1) !}\left(2 h-4^{-k}\right)+4 r \frac{\tilde{\gamma} m_{0}}{m_{1}} f \sum_{k=0}^{\infty}\left(\frac{-2 r}{m_{1}^{2}}\right)^{k} \frac{\left(b_{2}^{+}\right)^{k+1} b_{1}^{2 k+1}}{(2 k+1) !}
$$$$
+r\left(h-\frac{1}{2}\right) \sum_{k=0}^{\infty}\left(\frac{-2 r}{m_{1}^{2}}\right)^{k+1} \frac{\left(b_{2}^{+}\right)^{k+1} b_{1}^{2 k+2}}{(2 k+2) !}-2 r\left(b_{1}^{+}\right)^{2} \sum_{k=0}^{\infty}\left(\frac{-8 r}{m_{1}^{2}}\right)^{k} \frac{\left(b_{2}^{+}\right)^{k} b_{1}^{2 k+2}}{(2 k+2) !}
$$ 


$$
\begin{aligned}
& -2 r f^{+} f \sum_{k=0}^{\infty}\left(\frac{-2 r}{m_{1}^{2}}\right)^{k}\left\{\frac{\left(h-\frac{1}{2}\right)}{(2 k) !}+\frac{b_{1}^{+} b_{1}}{(2 k+1) !}\right\}\left(b_{2}^{+}\right)^{k} b_{1}^{2 k} \\
& +\frac{m_{0}^{2}-r\left(h^{2}-\frac{1}{4}\right)}{2} \sum_{k=0}^{\infty}\left(\frac{-8 r}{m_{1}^{2}}\right)^{k+1} \frac{\left(b_{2}^{+}\right)^{k+1} b_{1}^{2 k+2}}{(2 k+2) !} \\
& l_{1}^{\prime}=-m_{1} b_{1}^{+} b_{2}+\frac{m_{1}}{4} b_{1}^{+} \sum_{k=1}^{\infty}\left(\frac{-8 r}{m_{1}^{2}}\right)^{k}\left\{\frac{2 h-4^{-k}}{(2 k) !}+\frac{2 b_{1}^{+} b_{1}}{(2 k+1) !}\right\}\left(b_{2}^{+}\right)^{k-1} b_{1}^{2 k}+ \\
& +\frac{\tilde{\gamma} m_{0}}{4}\left(f^{+}-2 b_{2}^{+} f\right) \sum_{k=1}^{\infty}\left(\frac{-8 r}{m_{1}^{2}}\right)^{k} \frac{\left(b_{2}^{+}\right)^{k-1} b_{1}^{2 k}}{(2 k) !}\left(1-4^{-k}\right) \\
& +\frac{r\left(h-\frac{1}{2}\right)}{2 m_{1}} \sum_{k=0}^{\infty}\left(\frac{-2 r}{m_{1}^{2}}\right)^{k} \frac{\left(b_{2}^{+}\right)^{k} b_{1}^{2 k+1}}{(2 k+1) !}+\frac{m_{1}}{2} b_{1}^{+} f^{+} f \sum_{k=1}^{\infty}\left(\frac{-2 r}{m_{1}^{2}}\right)^{k} \frac{\left(b_{2}^{+}\right)^{k-1} b_{1}^{2 k}}{(2 k) !} \\
& -\frac{r\left(h-\frac{1}{2}\right)}{m_{1}} f^{+} f \sum_{k=0}^{\infty}\left(\frac{-2 r}{m_{1}^{2}}\right)^{k} \frac{\left(b_{2}^{+}\right)^{k} b_{1}^{2 k+1}}{(2 k+1) !}-\tilde{\gamma} m_{0} f \sum_{k=0}^{\infty}\left(\frac{-2 r}{m_{1}^{2}}\right)^{k} \frac{\left(b_{2}^{+}\right)^{k} b_{1}^{2 k}}{(2 k) !} \\
& +\frac{m_{0}^{2}-r\left(h^{2}-\frac{1}{4}\right)}{m_{1}} \sum_{k=0}^{\infty}\left(\frac{-8 r}{m_{1}^{2}}\right)^{k} \frac{\left(b_{2}^{+}\right)^{k} b_{1}^{2 k+1}}{(2 k+1) !} \\
& l_{2}^{\prime}=g_{0}^{\prime} b_{2}-b_{2}^{+} b_{2}^{2}-\frac{m_{0}^{2}-r\left(h^{2}-\frac{1}{4}\right)}{m_{1}^{2}} \sum_{k=0}^{\infty}\left(\frac{-8 r}{m_{1}^{2}}\right)^{k} \frac{\left(b_{2}^{+}\right)^{k} b_{1}^{2 k+2}}{(2 k+2) !} \\
& -\frac{r\left(h-\frac{1}{2}\right)}{2 m_{1}^{2}} \sum_{k=0}^{\infty}\left(\frac{-2 r}{m_{1}^{2}}\right)^{k} \frac{\left(b_{2}^{+}\right)^{k} b_{1}^{2 k+2}}{(2 k+2) !}+\frac{\tilde{\gamma} m_{0}}{m_{1}} f \sum_{k=0}^{\infty}\left(\frac{-2 r}{m_{1}^{2}}\right)^{k} \frac{\left(b_{2}^{+}\right)^{k} b_{1}^{2 k+1}}{(2 k+1) !} \\
& -\frac{\tilde{\gamma} m_{0}}{4 m_{1}}\left(f^{+}-2 b_{2}^{+} f\right) \sum_{k=1}^{\infty}\left(\frac{-8 r}{m_{1}^{2}}\right)^{k} \frac{\left(b_{2}^{+}\right)^{k-1} b_{1}^{2 k+1}}{(2 k+1) !}\left(1-4^{-k}\right) \\
& -\frac{1}{4} b_{1}^{+} \sum_{k=1}^{\infty}\left(\frac{-8 r}{m_{1}^{2}}\right)^{k}\left\{\frac{2 h-4^{-k}}{(2 k+1) !}+\frac{2 b_{1}^{+} b_{1}}{(2 k+2) !}\right\}\left(b_{2}^{+}\right)^{k-1} b_{1}^{2 k+1} \\
& -\frac{1}{2} f^{+} f \sum_{k=1}^{\infty}\left(\frac{-2 r}{m_{1}^{2}}\right)^{k}\left\{\frac{h-\frac{1}{2}}{(2 k) !}+\frac{b_{1}^{+} b_{1}}{(2 k+1) !}\right\}\left(b_{2}^{+}\right)^{k-1} b_{1}^{2 k} \text {. }
\end{aligned}
$$

In the above expressions, $h$ is an arbitrary dimensionless constant, while $m_{0}$ and $m_{1}$ are arbitrary constants with the dimension of mass. To obtain explicit expressions for the additional parts (56) - (62) in the Fock space, we have introduced two new pairs of bosonic and a new pair of fermionic creation and annihilation operators satisfying the standard commutation relations

$$
\left[b_{1}, b_{1}^{+}\right]=1, \quad\left[b_{2}, b_{2}^{+}\right]=1, \quad\left\{f, f^{+}\right\}=1 .
$$

The resulting additional parts of the operators possess all the necessary properties, in particular, the additional parts which correspond to Hermitian operators contain arbitrary parameters (see the footnote 5): the operator $t_{0}^{\prime}$ contains the arbitrary parameter $m_{0}$; the operator $g_{0}^{\prime}$ contains the arbitrary parameter $h$. The values of the arbitrary parameters $h$ and $m_{0}$ will be determined later by the condition that the correct equations of motion (12), or, equivalently, (45), be reproduced.

The massive parameter $m_{1}$ remains arbitrary and can be expressed in terms of other parameters of the theory,

$$
m_{1}=f(m, r) \neq 0 .
$$


This arbitrariness does not affect the equations for the basic vector (5).

Note that the additional parts do not obey the usual properties

$$
\begin{array}{lll}
\left(l_{0}^{\prime}\right)^{+} \neq l_{0}^{\prime}, & \left(l_{1}^{\prime}\right)^{+} \neq l_{1}^{\prime+}, & \left(l_{2}^{\prime}\right)^{+} \neq l_{2}^{\prime+}, \\
\left(t_{0}^{\prime}\right)^{+} \neq t_{0}^{\prime} & \left(t_{1}^{\prime}\right)^{+} \neq t_{1}^{\prime+},
\end{array}
$$

if one should use the standard rules of Hermitian conjugation for the new creation and annihilation operators,

$$
\left(b_{1}\right)^{+}=b_{1}^{+}, \quad\left(b_{2}\right)^{+}=b_{2}^{+}, \quad(f)^{+}=f^{+} .
$$

To restore the proper Hermitian conjugation properties for the additional parts, we change the scalar product in the Fock space $\mathcal{H}^{\prime}$ generated by the new creation and annihilation operators as follows:

$$
\left\langle\tilde{\Psi}_{1} \mid \Psi_{2}\right\rangle_{\text {new }}=\left\langle\tilde{\Psi}_{1}\left|K^{\prime}\right| \Psi_{2}\right\rangle
$$

for any vectors $\left|\Psi_{1}\right\rangle,\left|\Psi_{2}\right\rangle$ with some, yet unknown, operator $K^{\prime}$. This operator is determined by the condition that all of the operators of the algebra must have the proper Hermitian properties with respect to the new scalar product:

$$
\begin{array}{rlrl}
\left\langle\tilde{\Psi}_{1}\left|K^{\prime} l_{0}^{\prime}\right| \Psi_{2}\right\rangle & =\left\langle\tilde{\Psi}_{2}\left|K^{\prime} l_{0}^{\prime}\right| \Psi_{1}\right\rangle^{*}, & & \left\langle\tilde{\Psi}_{1}\left|K^{\prime} t_{0}^{\prime}\right| \Psi_{2}\right\rangle=\left\langle\tilde{\Psi}_{2}\left|K^{\prime} t_{0}^{\prime}\right| \Psi_{1}\right\rangle^{*}, \\
\left\langle\tilde{\Psi}_{1}\left|K^{\prime} l_{1}^{\prime}\right| \Psi_{2}\right\rangle=\left\langle\tilde{\Psi}_{2}\left|K^{\prime} l_{1}^{\prime+}\right| \Psi_{1}\right\rangle^{*}, & & \left\langle\tilde{\Psi}_{1}\left|K^{\prime} t_{1}^{\prime}\right| \Psi_{2}\right\rangle=\left\langle\tilde{\Psi}_{2}\left|K^{\prime} t_{1}^{\prime+}\right| \Psi_{1}\right\rangle^{*} \\
\left\langle\tilde{\Psi}_{1}\left|K^{\prime} l_{2}^{\prime}\right| \Psi_{2}\right\rangle=\left\langle\tilde{\Psi}_{2}\left|K^{\prime} l_{2}^{\prime+}\right| \Psi_{1}\right\rangle^{*}, & & \left\langle\tilde{\Psi}_{1}\left|K^{\prime} g_{0}^{\prime}\right| \Psi_{2}\right\rangle=\left\langle\tilde{\Psi}_{2}\left|K^{\prime} g_{0}^{\prime}\right| \Psi_{1}\right\rangle^{*}
\end{array}
$$

These relations permit one to determine the operator $K^{\prime}$ as follows:

$$
K^{\prime}=Z^{+} Z, \quad Z=\sum_{n_{1}, n_{2}=0}^{\infty} \sum_{s=0}^{1}\left(\frac{l_{1}^{\prime+}}{m_{1}}\right)^{n_{1}}\left(t_{1}^{\prime+}\right)^{s}\left(l_{2}^{\prime+}\right)^{n_{2}}|0\rangle_{V} \frac{1}{n_{1} ! n_{2} !}\langle 0| b_{1}^{n_{1}} f^{s} b_{2}^{n_{2}}
$$

where the auxiliary vector $|0\rangle_{V}$ obeys the relations

$$
\begin{aligned}
& t_{1}^{\prime}|0\rangle_{V}=l_{1}^{\prime}|0\rangle_{V}=l_{2}^{\prime}|0\rangle_{V}=0, \quad{ }_{V}\langle 0 \mid 0\rangle_{V}=1 \\
& t_{0}^{\prime}|0\rangle_{V}=\tilde{\gamma} m_{0}|0\rangle_{V}, \quad l_{0}^{\prime}|0\rangle_{V}=m_{0}^{2}|0\rangle_{V}, \quad g_{0}^{\prime}|0\rangle_{V}=h|0\rangle_{V} .
\end{aligned}
$$

For low numbers $n_{1}+n_{f}+2 n_{2}$, where $n_{i}$ are the numbers of "particles" associated with $b_{i}^{+}$, and $n_{f}$ is the number of "particles" associated with $f^{+}$, the operator $K^{\prime}$ reads

$$
\begin{gathered}
K^{\prime}=|0\rangle\left\langle 0\left|+\frac{m_{0}^{2}-r h\left(h-\frac{1}{2}\right)}{m_{1}^{2}} b_{1}^{+}\right| 0\right\rangle\left\langle 0\left|b_{1}-2 h f^{+}\right| 0\right\rangle\langle 0| f \\
+\frac{m_{0}}{m_{1}}\left(\tilde{\gamma} f^{+}|0\rangle\left\langle 0\left|b_{1}+b_{1}^{+}\right| 0\right\rangle\langle 0| f \tilde{\gamma}\right)+\ldots
\end{gathered}
$$

This expression for the operator $K^{\prime}$ will be used later in constructing of examples in section 7.

Thus, in this section, we have constructed the additional parts (56) $-(62)$ for the constraints, which obey all the requirements. In the next section, we determine the algebra of the enlarged constraints and construct a BRST operator corresponding to this algebra. 


\begin{tabular}{|c|c|c|c|c|c|c|c|c|c|}
\hline$[\downarrow, \rightarrow\}$ & $T_{0}$ & $T_{1}$ & $T_{1}^{+}$ & $L_{0}$ & $L_{1}$ & $L_{1}^{+}$ & $L_{2}$ & $L_{2}^{+}$ & $G_{0}$ \\
\hline$T_{0}$ & $-2 L_{0}$ & $2 L_{1}$ & $2 L_{1}^{+}$ & 0 & (176) & $-(77)$ & 0 & 0 & 0 \\
\hline$T_{1}$ & $2 L_{1}$ & $4 L_{2}$ & $-2 G_{0}$ & (78) & 0 & $-T_{0}$ & 0 & $-T_{1}^{+}$ & $T_{1}$ \\
\hline$T_{1}^{+}$ & $2 L_{1}^{+}$ & $-2 G_{0}$ & $4 L_{2}^{+}$ & $-(79)$ & $T_{0}$ & 0 & $T_{1}$ & 0 & $-T_{1}^{+}$ \\
\hline$L_{0}$ & 0 & $-(178)$ & (79) & 0 & -80 & (81) & 0 & 0 & 0 \\
\hline$L_{1}$ & $-(76)$ & 0 & $-T_{0}$ & (80) & 0 & (82) & 0 & $-L_{1}^{+}$ & $L_{1}$ \\
\hline$L_{1}^{+}$ & (177) & $T_{0}$ & 0 & -81 & -82 & 0 & $L_{1}$ & 0 & $-L_{1}^{+}$ \\
\hline$L_{2}$ & 0 & 0 & $-T_{1}$ & 0 & 0 & $-L_{1}$ & 0 & $G_{0}$ & $2 L_{2}$ \\
\hline$L_{2}^{+}$ & 0 & $T_{1}^{+}$ & 0 & 0 & $L_{1}^{+}$ & 0 & $-G_{0}$ & 0 & $-2 L_{2}^{+}$ \\
\hline$G_{0}$ & 0 & $-T_{1}$ & $T_{1}^{+}$ & 0 & $-L_{1}$ & $L_{1}^{+}$ & $-2 L_{2}$ & $2 L_{2}^{+}$ & 0 \\
\hline
\end{tabular}

Table 4: The algebra of the enlarged operators.

\section{Deformed algebra and BRST operator}

Let us turn to the algebra of the enlarged operators $\tilde{O}_{i}=\tilde{o}_{i}+o_{i}^{\prime}$. Since the algebra is quadratic, there exist different possibilities of operator ordering in the right-hand side of the commutation relations. Analogous situation takes place in the bosonic higher spin theory as well. In [33] we have studied dependence of BRST construction on ordering prescription for the quadratic constraint algebra and proved that different ordering prescriptions lead to equivalent Lagrangians and the same solution to the equations of motion. Since the constraint algebra in the fermionic case is analogous to the bosonic one, the same consequence about the final Lagrangians and the equations of motion will be valid also for the fermionic case. Therefore we are not going to study here all the possibilities of ordering prescriptions as this was done in [33], but choose only one of them, which corresponds to the supersymmetrized ordering for the constraints and lead to the expression for the BRST operator which has nonvanishing terms of the third degree in powers of ghosts 6 . The algebra of the enlarged operators corresponding to this BRST operator is given by Table 4, where

\footnotetext{
${ }^{6}$ Using our results in the bosonic case [33], we see that the BRST operators corresponding to different choices of the mentioned ordering of constraints will lead to equivalent Lagrangian formulations. At the same time, we note that for the purpose of constructing Lagrangians it is sufficient to find only one BRST operator with a some fixed ordering for the constraints in its commutation relations.
} 


$$
\begin{aligned}
& {\left[T_{0}, L_{1}\right\}=} r\left[\frac{1}{2} G_{0} T_{1}+\frac{1}{2} T_{1} G_{0}+T_{1}^{+} L_{2}+L_{2} T_{1}^{+}-g_{0}^{\prime} T_{1}-t_{1}^{\prime} G_{0}-2\left(t_{1}^{\prime+} L_{2}+l_{2}^{\prime} T_{1}^{+}\right)\right] \\
& {\left[L_{1}^{+}, T_{0}\right\}=} r\left[\frac{1}{2} T_{1}^{+} G_{0}+\frac{1}{2} G_{0} T_{1}^{+}+L_{2}^{+} T_{1}+T_{1} L_{2}^{+}-t_{1}^{\prime+} G_{0}-g_{0}^{\prime} T_{1}^{+}-2\left(l_{2}^{\prime+} T_{1}+t_{1}^{\prime} L_{2}^{+}\right)\right] \\
& {\left[T_{1}, L_{0}\right\}=} r\left[G_{0} T_{1}+T_{1} G_{0}+2 T_{1}^{+} L_{2}+2 L_{2} T_{1}^{+}-2\left(g_{0}^{\prime} T_{1}+t_{1}^{\prime} G_{0}\right)-4\left(t_{1}^{\prime+} L_{2}+l_{2}^{\prime} T_{1}^{+}\right)\right] \\
& {\left[L_{0}, T_{1}^{+}\right\}=} r\left[T_{1}^{+} G_{0}+G_{0} T_{1}^{+}+2 L_{2}^{+} T_{1}+2 T_{1} L_{2}^{+}-2\left(t_{1}^{\prime+} G_{0}+g_{0}^{\prime} T_{1}^{+}\right)-4\left(l_{2}^{\prime+} T_{1}+t_{1}^{\prime} L_{2}^{+}\right)\right] \\
& {\left[L_{1}, L_{0}\right\}=} r\left[G_{0} L_{1}+L_{1} G_{0}+2 L_{1}^{+} L_{2}+2 L_{2} L_{1}^{+}-2\left(l_{1}^{\prime} G_{0}+g_{0}^{\prime} L_{1}\right)-4\left(l_{1}^{\prime+} L_{2}+l_{2}^{\prime} L_{1}^{+}\right)\right] \\
& {\left[L_{0}, L_{1}^{+}\right\}=} r\left[L_{1}^{+} G_{0}+G_{0} L_{1}^{+}+2 L_{2}^{+} L_{1}+2 L_{1} L_{2}^{+}-2\left(l_{1}^{\prime+} G_{0}+g_{0}^{\prime} L_{1}^{+}\right)-4\left(l_{1}^{\prime} L_{2}^{+}+l_{2}^{\prime+} L_{1}\right)\right] \\
& {\left[L_{1}, L_{1}^{+}\right\}=} L_{0}+r\left\{G_{0}^{2}-2 L_{2}^{+} L_{2}-2 L_{2} L_{2}^{+}+\frac{3}{4} T_{1}^{+} T_{1}-\frac{3}{4} T_{1} T_{1}^{+}\right\} \\
& \quad+r\left\{-2 g_{0}^{\prime} G_{0}+4\left(l_{2}^{\prime+} L_{2}+l_{2}^{\prime} L_{2}^{+}\right)-\frac{3}{2}\left(t_{1}^{\prime+} T_{1}-t_{1}^{\prime} T_{1}^{+}\right)\right\}
\end{aligned}
$$

The construction of a nilpotent fermionic BRST operator for a nonlinear superalgebra is based on the same principles as those developed in [28,33] (for a general consideration of operator BFV quantization, see the reviews [25]). The BRST operator constructed on a basis of the algebra given by Table 4 can be calculated with the help of the $(\mathcal{C P})$-ordering of the ghost coordinate $\mathcal{C}^{i}$ and momenta $\mathcal{P}_{i}$ operators:

$$
\begin{aligned}
Q^{\prime}= & q_{0} T_{0}+q_{1}^{+} T_{1}+q_{1} T_{1}^{+}+\eta_{0} L_{0}+\eta_{1}^{+} L_{1}+\eta_{1} L_{1}^{+}+\eta_{2}^{+} L_{2}+\eta_{2} L_{2}^{+}+\eta_{G} G_{0} \\
& +i\left(\eta_{1}^{+} q_{1}-\eta_{1} q_{1}^{+}\right) p_{0}-i\left(\eta_{G} q_{1}+\eta_{2} q_{1}^{+}\right) p_{1}^{+}+i\left(\eta_{G} q_{1}^{+}+\eta_{2}^{+} q_{1}\right) p_{1} \\
& +\left(q_{0}^{2}-\eta_{1}^{+} \eta_{1}\right) \mathcal{P}_{0}+\left(2 q_{1} q_{1}^{+}-\eta_{2}^{+} \eta_{2}\right) \mathcal{P}_{G}+\left(\eta_{G} \eta_{1}^{+}+\eta_{2}^{+} \eta_{1}-2 q_{0} q_{1}^{+}\right) \mathcal{P}_{1} \\
& +\left(\eta_{1} \eta_{G}+\eta_{1}^{+} \eta_{2}-2 q_{0} q_{1}\right) \mathcal{P}_{1}^{+}+2\left(\eta_{G} \eta_{2}^{+}-q_{1}^{+2}\right) \mathcal{P}_{2}+2\left(\eta_{2} \eta_{G}-q_{1}^{2}\right) \mathcal{P}_{2}^{+} \\
+ & r\left(q_{0} \eta_{1}^{+}+2 q_{1}^{+} \eta_{0}\right)\left[\frac{i}{2} G_{0} p_{1}+\frac{1}{2} T_{1} \mathcal{P}_{G}+T_{1}^{+} \mathcal{P}_{2}+i L_{2} p_{1}^{+}-t_{1}^{\prime} \mathcal{P}_{G}-i g_{0}^{\prime} p_{1}-2\left(i l_{2}^{\prime} p_{1}^{+}+t_{1}^{\prime+} \mathcal{P}_{2}\right)\right] \\
+ & r\left(q_{0} \eta_{1}+2 q_{1} \eta_{0}\right)\left[-\frac{i}{2} G_{0} p_{1}^{+}-\frac{1}{2} T_{1}^{+} \mathcal{P}_{G}-T_{1} \mathcal{P}_{2}^{+}-i L_{2}^{+} p_{1}+t_{1}^{\prime+} \mathcal{P}_{G}+i g_{0}^{\prime} p_{1}^{+}+2\left(i l_{2}^{\prime+} p_{1}+t_{1}^{\prime} \mathcal{P}_{2}^{+}\right)\right] \\
& +2 r \eta_{0} \eta_{1}^{+}\left[\frac{1}{2} G_{0} \mathcal{P}_{1}+\frac{1}{2} L_{1} \mathcal{P}_{G}+L_{1}^{+} \mathcal{P}_{2}+L_{2} \mathcal{P}_{1}^{+}-l_{1}^{\prime} \mathcal{P}_{G}-g_{0}^{\prime} \mathcal{P}_{1}-2\left(l_{1}^{\prime+} \mathcal{P}_{2}+l_{2}^{\prime} \mathcal{P}_{1}^{+}\right)\right] \\
& -2 r \eta_{0} \eta_{1}\left[\frac{1}{2} G_{0} \mathcal{P}_{1}^{+}+\frac{1}{2} L_{1}^{+} \mathcal{P}_{G}+L_{1} \mathcal{P}_{2}^{+}+L_{2}^{+} \mathcal{P}_{1}-l_{1}^{\prime+} \mathcal{P}_{G}-g_{0}^{\prime} \mathcal{P}_{1}^{+}-2\left(l_{2}^{\prime+} \mathcal{P}_{1}+l_{1}^{\prime} \mathcal{P}_{2}^{+}\right)\right] \\
& -r \eta_{1} \eta_{1}^{+}\left[2 L_{2}^{+} \mathcal{P}_{2}+2 L_{2} \mathcal{P}_{2}^{+}-G_{0} \mathcal{P}_{G}-\frac{3 i}{4} T_{1}^{+} p_{1}+\frac{3 i}{4} T_{1} p_{1}^{+}\right] \\
+ & r \eta_{1} \eta_{1}^{+}\left[4\left(l_{2}^{\prime+} \mathcal{P}_{2}+l_{2}^{\prime} \mathcal{P}_{2}^{+}\right)-2 g_{0}^{\prime} \mathcal{P}_{G}-\frac{3 i}{2}\left(t_{1}^{\prime+} p_{1}-t_{1}^{\prime} p_{1}^{+}\right)\right] \\
+ & r^{2} \eta_{0} \eta_{1} \eta_{1}^{+}\left[G_{0}\left(p_{1}^{+} p_{1}+2 \mathcal{P}_{2}^{+} \mathcal{P}_{2}\right)+L_{2}^{+} p_{1}^{2}+L_{2}\left(p_{1}^{+}\right)^{2}+\frac{i}{2}\left(T_{1}^{+} p_{1}^{+} \mathcal{P}_{2}+T_{1} p_{1} \mathcal{P}_{2}^{+}\right)\right. \\
& \left.\quad+\frac{i}{4}\left(T_{1}^{+} p_{1}+T_{1} p_{1}^{+}\right) \mathcal{P}_{G}-2\left(L_{2} \mathcal{P}_{2}^{+}-L_{2}^{+} \mathcal{P}_{2}\right) \mathcal{P}_{G}\right]
\end{aligned}
$$

Here, $q_{0}, q_{1}, q_{1}^{+}$and $\eta_{0}, \eta_{1}^{+}, \eta_{1}, \eta_{2}^{+}, \eta_{2}, \eta_{G}$ are, respectively, the bosonic and fermionic ghost "coordinates" corresponding to their canonically conjugate ghost "momenta" $p_{0}, p_{1}^{+}, p_{1}, \mathcal{P}_{0}$, $\mathcal{P}_{1}, \mathcal{P}_{1}^{+}, \mathcal{P}_{2}, \mathcal{P}_{2}^{+}, \mathcal{P}_{G}$. They obey the (anti)commutation relations

$$
\begin{gathered}
\left\{\eta_{1}, \mathcal{P}_{1}^{+}\right\}=\left\{\mathcal{P}_{1}, \eta_{1}^{+}\right\}=\left\{\eta_{2}, \mathcal{P}_{2}^{+}\right\}=\left\{\mathcal{P}_{2}, \eta_{2}^{+}\right\}=\left\{\eta_{0}, \mathcal{P}_{0}\right\}=\left\{\eta_{G}, \mathcal{P}_{G}\right\}=1, \\
{\left[q_{0}, p_{0}\right]=\left[q_{1}, p_{1}^{+}\right]=\left[q_{1}^{+}, p_{1}\right]=i}
\end{gathered}
$$

and possess the standard ghost number distribution, $g h\left(\mathcal{C}^{i}\right)=-g h\left(\mathcal{P}_{i}\right)=1$, providing the property $g h\left(\tilde{Q}^{\prime}\right)=1$. 
The resulting BRST operator $Q^{\prime}$ is Hermitian with respect to the scalar product $\langle\mid\rangle$ in the tensor product of the Fock spaces $\mathcal{H}_{t o t}=\mathcal{H}^{\prime} \otimes \mathcal{H} \otimes \mathcal{H}_{g h}$, which is constructed as the direct product of the scalar products on $\mathcal{H}, \mathcal{H}^{\prime}$ and $\mathcal{H}_{g h}$,

$$
\left\langle\tilde{\Psi}_{1}\left|Q^{\prime+} K\right| \Psi_{2}\right\rangle=\left\langle\tilde{\Psi}_{1}\left|K Q^{\prime}\right| \Psi_{2}\right\rangle
$$

with the operator $K$ defined in $\mathcal{H}_{\text {tot }}$ and being the tensor product of the operator $K^{\prime}$ and the unit operators $\hat{1}, \hat{1}_{g h}$,

$$
K=K^{\prime} \otimes \hat{1} \otimes \hat{1}_{g h} .
$$

Thus, we have constructed the Hermitian BRST operator. In the next section, this operator is applied to construct Lagrangians of fermionic higher spin fields in the AdS space.

\section{Construction of Lagrangians}

In this section, we construct Lagrangians of fermionic massive higher spin fields in the AdS space. This construction goes along the lines of $[29,32]$. We should first extract the dependence of the BRST operator $Q^{\prime}(\underline{83})$ on the ghosts $\eta_{G}, \mathcal{P}_{G}$,

$$
Q^{\prime}=Q+\eta_{G}(\sigma+h)+\mathcal{B P}_{G},
$$

where

$$
\begin{aligned}
& Q= q_{0} T_{0}+q_{1}^{+} T_{1}+q_{1} T_{1}^{+}+\eta_{0} L_{0}+\eta_{1}^{+} L_{1}+\eta_{1} L_{1}^{+}+\eta_{2}^{+} L_{2}+\eta_{2} L_{2}^{+} \\
&+i\left(\eta_{1}^{+} q_{1}-\eta_{1} q_{1}^{+}\right) p_{0}-i \eta_{2} q_{1}^{+} p_{1}^{+}+i \eta_{2}^{+} q_{1} p_{1}+\left(q_{0}^{2}-\eta_{1}^{+} \eta_{1}\right) \mathcal{P}_{0}+\left(\eta_{2}^{+} \eta_{1}-2 q_{0} q_{1}^{+}\right) \mathcal{P}_{1} \\
&+\left(\eta_{1}^{+} \eta_{2}-2 q_{0} q_{1}\right) \mathcal{P}_{1}^{+}-2 q_{1}^{+2} \mathcal{P}_{2}-2 q_{1}^{2} \mathcal{P}_{2}^{+} \\
&+ r\left(q_{0} \eta_{1}^{+}+2 q_{1}^{+} \eta_{0}\right)\left[\frac{i}{2} G_{0} p_{1}+T_{1}^{+} \mathcal{P}_{2}+i L_{2} p_{1}^{+}-i g_{0}^{\prime} p_{1}-2\left(i l_{2}^{\prime} p_{1}^{+}+t_{1}^{\prime+} \mathcal{P}_{2}\right)\right] \\
&+ r\left(q_{0} \eta_{1}+2 q_{1} \eta_{0}\right)\left[-\frac{i}{2} G_{0} p_{1}^{+}-T_{1} \mathcal{P}_{2}^{+}-i L_{2}^{+} p_{1}+i g_{0}^{\prime} p_{1}^{+}+2\left(i l_{2}^{\prime+} p_{1}+t_{1}^{\prime} \mathcal{P}_{2}^{+}\right)\right] \\
&+2 r \eta_{0} \eta_{1}^{+}\left[\frac{1}{2} G_{0} \mathcal{P}_{1}+L_{1}^{+} \mathcal{P}_{2}+L_{2} \mathcal{P}_{1}^{+}-g_{0}^{\prime} \mathcal{P}_{1}-2\left(l_{1}^{\prime+} \mathcal{P}_{2}+l_{2}^{\prime} \mathcal{P}_{1}^{+}\right)\right] \\
&-2 r \eta_{0} \eta_{1}\left[\frac{1}{2} G_{0} \mathcal{P}_{1}^{+}+L_{1} \mathcal{P}_{2}^{+}+L_{2}^{+} \mathcal{P}_{1}-g_{0}^{\prime} \mathcal{P}_{1}^{+}-2\left(l_{2}^{\prime+} \mathcal{P}_{1}+l_{1}^{\prime} \mathcal{P}_{2}^{+}\right)\right] \\
&-r \eta_{1} \eta_{1}^{+}\left[2 L_{2}^{+} \mathcal{P}_{2}+2 L_{2} \mathcal{P}_{2}^{+}-\frac{3 i}{4} T_{1}^{+} p_{1}+\frac{3 i}{4} T_{1} p_{1}^{+}\right] \\
&+r \eta_{1} \eta_{1}^{+}\left[4\left(l_{2}^{\prime+} \mathcal{P}_{2}+l_{2}^{\prime} \mathcal{P}_{2}^{+}\right)-\frac{3 i}{2}\left(t_{1}^{\prime+} p_{1}-t_{1}^{\prime} p_{1}^{+}\right)\right] \\
& r^{2} \eta_{0} \eta_{1} \eta_{1}^{+}\left[G_{0}\left(p_{1}^{+} p_{1}+2 \mathcal{P}_{2}^{+} \mathcal{P}_{2}\right)+L_{2}^{+} p_{1}^{2}+L_{2}\left(p_{1}^{+}\right)^{2}+\frac{i}{2}\left(T_{1}^{+} p_{1}^{+} \mathcal{P}_{2}+T_{1} p_{1} \mathcal{P}_{2}^{+}\right)\right] \\
& \sigma \quad-a_{\mu}^{+} a^{\mu}+\frac{d}{2}+b_{1}^{+} b_{1}+2 b_{2}^{+} b_{2}+f^{+} f \\
& \quad-i q_{1} p_{1}^{+}+i q_{1}^{+} p_{1}+\eta_{1}^{+} \mathcal{P}_{1}-\eta_{1} \mathcal{P}_{1}^{+}+2 \eta_{2}^{+} \mathcal{P}_{2}-2 \eta_{2} \mathcal{P}_{2}^{+} \\
& \quad=
\end{aligned}
$$

meanwhile the explicit expression for the operator $\mathcal{B}$ is not essential.

Next, following the procedure of $[29,32]$, we choose the following representation of the Hilbert space:

$$
\left(p_{0}, q_{1}, p_{1}, \mathcal{P}_{0}, \mathcal{P}_{G}, \eta_{1}, \mathcal{P}_{1}, \eta_{2}, \mathcal{P}_{2}\right)|0\rangle=0
$$


and suppose that the vectors and gauge parameters do not depend on $\eta_{G}$,

$$
\begin{gathered}
|\chi\rangle=\sum_{k_{i}}\left(q_{0}\right)^{k_{1}}\left(q_{1}^{+}\right)^{k_{2}}\left(p_{1}^{+}\right)^{k_{3}}\left(\eta_{0}\right)^{k_{4}}\left(f^{+}\right)^{k_{5}}\left(\eta_{1}^{+}\right)^{k_{6}}\left(\mathcal{P}_{1}^{+}\right)^{k_{7}}\left(\eta_{2}^{+}\right)^{k_{8}}\left(\mathcal{P}_{2}^{+}\right)^{k_{9}}\left(b_{1}^{+}\right)^{k_{10}}\left(b_{2}^{+}\right)^{k_{11}} \times \\
\times a^{+\mu_{1}} \cdots a^{+\mu_{k_{0}}} \chi_{\mu_{1} \cdots \mu_{k_{0}}}^{k_{1} \cdots k_{11}}(x)|0\rangle .
\end{gathered}
$$

The sum in (91) is taken over $k_{0}, k_{1}, k_{2}, k_{3}, k_{10}, k_{11}$, running from 0 to infinity, and over $k_{4}, k_{5}, k_{6}, k_{7}, k_{8}, k_{9}$, running from 0 to 1 . Then, we derive from the equations that determine the physical vector, $Q^{\prime}|\chi\rangle=0$, as well as from the reducible gauge transformations, $\delta|\chi\rangle=Q^{\prime}|\Lambda\rangle$, a sequence of relations:

$$
\begin{array}{lll}
Q|\chi\rangle=0, & (\sigma+h)|\chi\rangle=0, & (\varepsilon, g h)(|\chi\rangle)=(1,0), \\
\delta|\chi\rangle=Q|\Lambda\rangle, & (\sigma+h)|\Lambda\rangle=0, & (\varepsilon, g h)(|\Lambda\rangle)=(0,-1), \\
\delta|\Lambda\rangle=Q\left|\Lambda^{(1)}\right\rangle, & (\sigma+h)\left|\Lambda^{(1)}\right\rangle=0, & (\varepsilon, g h)\left(\left|\Lambda^{(1)}\right\rangle\right)=(1,-2), \\
\delta\left|\Lambda^{(i-1)}\right\rangle=Q\left|\Lambda^{(i)}\right\rangle, & (\sigma+h)\left|\Lambda^{(i)}\right\rangle=0, & (\varepsilon, g h)\left(\left|\Lambda^{(i)}\right\rangle\right)=(i,-i-1) .
\end{array}
$$

The middle equation in (92) presents the equations for the possible values of $h$,

$$
-h=n+\frac{d-4}{2}
$$

with $n$ being related to spin, $s=n+1 / 2$. By fixing the value of spin, we also fix the parameter $h$, according to (96). Having fixed a value of $h$, we must substitute it into each of the expressions (92)-(95); see [29] for more details.

As a next step, we have to extract the zero-mode ghosts from the operator $Q$ (88). This operator has the structure

$$
Q=q_{0} \tilde{T}_{0}+\eta_{0} \tilde{L}_{0}+i\left(\eta_{1}^{+} q_{1}-\eta_{1} q_{1}^{+}\right) p_{0}+\left(q_{0}^{2}-\eta_{1}^{+} \eta_{1}\right) \mathcal{P}_{0}+\Delta Q
$$

where

$$
\begin{aligned}
\tilde{T}_{0}= & T_{0}-2 q_{1}^{+} \mathcal{P}_{1}-2 q_{1} \mathcal{P}_{1}^{+} \\
+ & r \eta_{1}^{+}\left[\frac{i}{2} G_{0} p_{1}+T_{1}^{+} \mathcal{P}_{2}+i L_{2} p_{1}^{+}-i g_{0}^{\prime} p_{1}-2\left(i l_{2}^{\prime} p_{1}^{+}+t_{1}^{\prime+} \mathcal{P}_{2}\right)\right] \\
+ & r \eta_{1}\left[-\frac{i}{2} G_{0} p_{1}^{+}-T_{1} \mathcal{P}_{2}^{+}-i L_{2}^{+} p_{1}+i g_{0}^{\prime} p_{1}^{+}+2\left(i l_{2}^{\prime+} p_{1}+t_{1}^{\prime} \mathcal{P}_{2}^{+}\right)\right] \\
\tilde{L}_{0}= & L_{0}+2 r q_{1}^{+}\left[\frac{i}{2} G_{0} p_{1}+T_{1}^{+} \mathcal{P}_{2}+i L_{2} p_{1}^{+}-i g_{0}^{\prime} p_{1}-2\left(i l_{2}^{\prime} p_{1}^{+}+t_{1}^{\prime+} \mathcal{P}_{2}\right)\right] \\
+ & 2 r q_{1}\left[-\frac{i}{2} G_{0} p_{1}^{+}-T_{1} \mathcal{P}_{2}^{+}-i L_{2}^{+} p_{1}+i g_{0}^{\prime} p_{1}^{+}+2\left(i l_{2}^{\prime+} p_{1}+t_{1}^{\prime} \mathcal{P}_{2}^{+}\right)\right] \\
+ & 2 r \eta_{1}^{+}\left[\frac{1}{2} G_{0} \mathcal{P}_{1}+L_{1}^{+} \mathcal{P}_{2}+L_{2} \mathcal{P}_{1}^{+}-g_{0}^{\prime} \mathcal{P}_{1}-2\left(l_{1}^{\prime+} \mathcal{P}_{2}+l_{2}^{\prime} \mathcal{P}_{1}^{+}\right)\right] \\
- & 2 r \eta_{1}\left[\frac{1}{2} G_{0} \mathcal{P}_{1}^{+}+L_{1} \mathcal{P}_{2}^{+}+L_{2}^{+} \mathcal{P}_{1}-g_{0}^{\prime} \mathcal{P}_{1}^{+}-2\left(l_{2}^{\prime+} \mathcal{P}_{1}+l_{1}^{\prime} \mathcal{P}_{2}^{+}\right)\right] \\
+ & r^{2} \eta_{1} \eta_{1}^{+}\left[G_{0}\left(p_{1}^{+} p_{1}+2 \mathcal{P}_{2}^{+} \mathcal{P}_{2}\right)+L_{2}^{+} p_{1}^{2}+L_{2}\left(p_{1}^{+}\right)^{2}+\frac{i}{2}\left(T_{1}^{+} p_{1}^{+} \mathcal{P}_{2}+T_{1} p_{1} \mathcal{P}_{2}^{+}\right)\right] \\
\quad & q_{1}^{+} T_{1}+q_{1} T_{1}^{+}+\eta_{1}^{+} L_{1}+\eta_{1} L_{1}^{+}+\eta_{2}^{+} L_{2}+\eta_{2} L_{2}^{+}-i \eta_{2} q_{1}^{+} p_{1}^{+}+i \eta_{2}^{+} q_{1} p_{1} \\
& +\eta_{2}^{+} \eta_{1} \mathcal{P}_{1}+\eta_{1}^{+} \eta_{2} \mathcal{P}_{1}^{+}-2 q_{1}^{+2} \mathcal{P}_{2}-2 q_{1}^{2} \mathcal{P}_{2}^{+} \\
& \quad r \eta_{1} \eta_{1}^{+}\left[2 L_{2}^{+} \mathcal{P}_{2}+2 L_{2} \mathcal{P}_{2}^{+}-\frac{3 i}{4} T_{1}^{+} p_{1}+\frac{3 i}{4} T_{1} p_{1}^{+}\right] \\
& +r \eta_{1} \eta_{1}^{+}\left[4\left(l_{2}^{\prime+} \mathcal{P}_{2}+l_{2}^{\prime} \mathcal{P}_{2}^{+}\right)-\frac{3 i}{2}\left(t_{1}^{\prime+} p_{1}-t_{1}^{\prime} p_{1}^{+}\right)\right]
\end{aligned}
$$


Here, $\tilde{T}_{0}, \tilde{L}_{0}, \Delta Q$ are independent of $q_{0}, p_{0}, \eta_{0}, \mathcal{P}_{0}$. We also expand the state vector and gauge parameters in powers of the zero-mode ghosts:

$$
\begin{aligned}
|\chi\rangle & =\sum_{k=0}^{\infty} q_{0}^{k}\left(\left|\chi_{0}^{k}\right\rangle+\eta_{0}\left|\chi_{1}^{k}\right\rangle\right), & & g h\left(\left|\chi_{m}^{k}\right\rangle\right)=-(m+k), \\
\left|\Lambda^{(i)}\right\rangle & =\sum_{k=0}^{\infty} q_{0}^{k}\left(\left|\Lambda_{0}^{(i) k}\right\rangle+\eta_{0}\left|\Lambda_{1}^{(i) k}\right\rangle\right), & & g h\left(\left|\Lambda_{m}^{(i) k}\right\rangle\right)=-(i+k+m+1) .
\end{aligned}
$$

Following the procedure of [29], we get rid of all the fields except two, $\left|\chi_{0}^{0}\right\rangle,\left|\chi_{0}^{1}\right\rangle$, and, hence, relations (92)-(95), with allowance for (97), (101), (102), yield two independent equations for these fields:

$$
\begin{aligned}
& \Delta Q\left|\chi_{0}^{0}\right\rangle+\frac{1}{2}\left\{\tilde{T}_{0}, \eta_{1}^{+} \eta_{1}\right\}\left|\chi_{0}^{1}\right\rangle=0, \\
& \tilde{T}_{0}\left|\chi_{0}^{0}\right\rangle+\Delta Q\left|\chi_{0}^{1}\right\rangle=0
\end{aligned}
$$

where $\{A, B\}=A B+B A$ for any quantities $A, B$.

Next, due to the fact that the operators $Q, \tilde{T}_{0}, \eta_{1}^{+} \eta_{1}$ commute with $\sigma$, we derive from (103), (104) the equations of motion for the fields with a fixed value of spin:

$$
\begin{aligned}
& \Delta Q\left|\chi_{0}^{0}\right\rangle_{n}+\frac{1}{2}\left\{\tilde{T}_{0}, \eta_{1}^{+} \eta_{1}\right\}\left|\chi_{0}^{1}\right\rangle_{n}=0, \\
& \tilde{T}_{0}\left|\chi_{0}^{0}\right\rangle_{n}+\Delta Q\left|\chi_{0}^{1}\right\rangle_{n}=0 .
\end{aligned}
$$

where the fields $\left|\chi_{0}^{0}\right\rangle,\left|\chi_{0}^{1}\right\rangle$ are assumed to obey the relations

$$
\sigma\left|\chi_{0}^{0}\right\rangle_{n}=(n+(d-4) / 2)\left|\chi_{0}^{0}\right\rangle_{n}, \quad \sigma\left|\chi_{0}^{1}\right\rangle_{n}=(n+(d-4) / 2)\left|\chi_{0}^{1}\right\rangle_{n} .
$$

The field equations (105), (106) are Lagrangian ones and can be deduced from the following action:7

$$
\begin{aligned}
\mathcal{S}_{n}= & { }_{n}\left\langle\tilde{\chi}_{0}^{0}\left|K_{n} \tilde{T}_{0}\right| \chi_{0}^{0}\right\rangle_{n}+\frac{1}{2}{ }_{n}\left\langle\tilde{\chi}_{0}^{1}\left|K_{n}\left\{\tilde{T}_{0}, \eta_{1}^{+} \eta_{1}\right\}\right| \chi_{0}^{1}\right\rangle_{n} \\
& +{ }_{n}\left\langle\tilde{\chi}_{0}^{0}\left|K_{n} \Delta Q\right| \chi_{0}^{1}\right\rangle_{n}+{ }_{n}\left\langle\tilde{\chi}_{0}^{1}\left|K_{n} \Delta Q\right| \chi_{0}^{0}\right\rangle_{n},
\end{aligned}
$$

where the standard scalar product for the creation and annihilation operators is assumed, and the operator $K_{n}$ is the operator $K(\underline{86})$, where the following substitution is made: $h \rightarrow$ $-(n+(d-4) / 2)$.

The equations of motion (105), (106) and the action (108) are invariant with respect to the gauge transformations

$$
\begin{aligned}
\delta\left|\chi_{0}^{0}\right\rangle_{n} & =\Delta Q\left|\Lambda_{0}^{0}\right\rangle_{n}+\frac{1}{2}\left\{\tilde{T}_{0}, \eta_{1}^{+} \eta_{1}\right\}\left|\Lambda_{0}^{1}\right\rangle_{n} \\
\delta\left|\chi_{0}^{1}\right\rangle_{n} & =\tilde{T}_{0}\left|\Lambda_{0}^{0}\right\rangle_{n}+\Delta Q\left|\Lambda_{0}^{1}\right\rangle_{n}
\end{aligned}
$$

which are reducible, with the gauge parameters $\left|\Lambda_{0}^{(i) j}\right\rangle_{n}, j=0,1$ subject to the same conditions as those for $\left|\chi_{0}^{j}\right\rangle_{n}$ in (107),

$$
\begin{aligned}
\delta\left|\Lambda_{0}^{(i) 0}\right\rangle_{n} & =\Delta Q\left|\Lambda^{(i+1) 0}\right\rangle_{n}+\frac{1}{2}\left\{\tilde{T}_{0}, \eta_{1}^{+} \eta_{1}\right\}\left|\Lambda_{0}^{(i+1) 1}\right\rangle_{n}, & & \left|\Lambda_{0}^{(0) 0}\right\rangle_{n}=\left|\Lambda_{0}^{0}\right\rangle_{n}, \\
\delta\left|\Lambda_{0}^{(i) 1}\right\rangle_{n} & =\tilde{T}_{0}\left|\Lambda^{(i+1) 0}{ }_{0}\right\rangle_{n}+\Delta Q\left|\Lambda^{(i+1) 1}\right\rangle_{n}, & \left|\Lambda_{0}^{(0) 1}\right\rangle_{n} & =\left|\Lambda_{0}^{1}\right\rangle_{n},
\end{aligned}
$$

\footnotetext{
${ }^{7}$ The action is defined, as usual, up to an overall factor.
} 
and with a finite number of reducibility stages at $i_{\max }=n-1$ for spin $s=n+1 / 2$.

We now determine the value of the arbitrary parameter $m_{0}$, using the condition that the equations (45) for the basic vector $|\Phi\rangle$ (5) be reproduced. To this end, it is necessary that conditions (45) be implied by Eqs. (105), (106). Note that the general vector $\left|\chi_{0}^{0}\right\rangle_{n}$ includes the basic vector $|\Phi\rangle(\underline{5})$, namely,

$$
\left|\chi_{0}^{0}\right\rangle_{n}=|\Phi\rangle_{n}+\left|\Phi_{A}\right\rangle_{n},\left.\quad\left|\Phi_{A}\right\rangle_{n}\right|_{\mathcal{C}=\mathcal{P}=b_{1}^{+}=b_{2}^{+}=f^{+}=0}=0
$$

In the next section, we shall demonstrate that due to the gauge transformations and a part of the equations of motion the vector $\left|\Phi_{A}\right\rangle_{n}$ can be completely removed, so that the resulting equations of motion have the form

$$
T_{0}|\Phi\rangle_{n}=\left(t_{0}+\tilde{\gamma} m_{0}\right)|\Phi\rangle_{n}=0, \quad t_{1}|\Phi\rangle_{n}=0
$$

The above relations permit one to determine the parameter $m_{0}$ in a unique way, as follows:

$$
m_{0}=m-r^{\frac{1}{2}} h=m+r^{\frac{1}{2}}(n+(d-4) / 2) .
$$

It should be noted that $m_{0}$ of the present article is the AdS mass $m_{D}$ of [34] corresponding to "the most-used definition of mass" (for more discussions of this point, see [34]).

In the next section, we shall demonstrate that the action actually reproduces the correct equations of motion (12). Thus, we have constructed Lagrangians for fermionic fields of any fixed spin using the BRST approach 8 .

\section{Reduction to the initial irreducible relations}

Let us show that the equations of motion (1), (2) [or equivalently (45)] can be obtained from the Lagrangian (108) after gauge-fixing and removing the auxiliary fields by using a part of the equations of motion. Let us start with gauge-fixing.

\subsection{Gauge-fixing}

Let us consider a field of spin $s=n+1 / 2$. Then we have a reducible gauge theory with $n-1$ reducibility stages. Due to restriction (107) and the ghost number restriction [see the right-hand formulae in (102)], the lowest-stage gauge parameters have the form

$$
\begin{aligned}
\left|\Lambda_{0}^{(n-1) 0}\right\rangle_{n} & =\left(p_{1}^{+}\right)^{n-1}\left\{\mathcal{P}_{1}^{+}|\lambda\rangle_{0}+p_{1}^{+}\left|\lambda_{1}\right\rangle_{0}\right\} \\
\left|\Lambda_{0}^{(n-1) 1}\right\rangle_{n} & \equiv 0
\end{aligned}
$$

with the subscripts of the state vectors being associated with the eigenvalues of the corresponding state vectors (107). It can be verified directly that one can eliminate the dependence on the ghost $\mathcal{P}_{2}^{+}$from the gauge function $\left|\Lambda_{0}^{(n-2) 0}\right\rangle$ of the $(n-2)$-th stage (the gauge function $\left|\Lambda_{0}^{(n-2) 1}\right\rangle$ has no $\mathcal{P}_{2}^{+}$dependence). It is then possible to verify that one can remove the dependence of $\left|\Lambda^{(n-3) 0}\right\rangle,\left|\Lambda^{(n-3)}{ }_{0}^{1}\right\rangle$ on $\mathcal{P}_{2}^{+}$with the help of the remaining gauge parameters (which do not depend on $\left.\mathcal{P}_{2}^{+}\right)\left|\Lambda_{0}^{(n-2) 0}\right\rangle,\left|\Lambda_{0}^{(n-2) 1}\right\rangle$.

\footnotetext{
${ }^{8}$ The construction of a Lagrangian describing the propagation of all fermionic fields in the AdS space simultaneously is analogous to the case of the flat space [29] and we do not consider it here. We only note that the necessary condition for resolving this problem is to replace in $Q^{\prime}, Q, K$ the parameter $-h$ by the operator $\sigma$ in an appropriate way and discard the condition (107) for the fields and gauge parameters.
} 
We now suppose that we have removed the dependence on $\mathcal{P}_{2}^{+}$from the gauge functions of the $(i+1)$-th stage $\left|\Lambda_{0}^{(i+1) j}\right\rangle, j=0,1$, i.e., we have $\eta_{2}\left|\Lambda_{0}^{(i+1) j}\right\rangle=0$. Let us show that these restricted gauge functions can be used to remove the dependence on $\mathcal{P}_{2}^{+}$from the gauge functions $\left|\Lambda_{0}^{(i) j}\right\rangle$. We introduce the following notation for the gauge parameters, related to their decomposition in ghosts $\mathcal{P}_{1}^{+}, \mathcal{P}_{2}^{+}$:

$$
\left|\Lambda_{0}^{(i) j}\right\rangle=\left|\Lambda_{00}^{(i) j}\right\rangle+\mathcal{P}_{1}^{+}\left|\Lambda_{01}^{(i) j}\right\rangle+\mathcal{P}_{2}^{+}\left|\Lambda_{02}^{(i) j}\right\rangle+\mathcal{P}_{1}^{+} \mathcal{P}_{2}^{+}\left|\Lambda_{03}^{(i) j}\right\rangle
$$

Here and elsewhere, we omit the subscripts of the vectors which are associated with the eigenvalues of the operator $\sigma$ (107). Then, using (111) and (112), we find a gauge transformation for $\left|\Lambda_{02}^{(i) j}\right\rangle$ and $\left|\Lambda_{03}^{(i) j}\right\rangle$, being coefficients at $\mathcal{P}_{2}^{+}$,

$$
\begin{aligned}
& \delta\left|\Lambda_{02}^{(i) 0}\right\rangle=-2 q_{1}^{2}\left|\Lambda_{00}^{(i+1) 0}\right\rangle+2 r \eta_{1}^{+}\left(L_{2}-2 l_{2}^{\prime}\right)\left|\Lambda_{01}^{(i+1) 0}\right\rangle, \\
& \delta\left|\Lambda_{03}^{(i) 0}\right\rangle=2 q_{1}^{2}\left|\Lambda_{01}^{(i+1) 0}\right\rangle, \\
& \delta\left|\Lambda_{02}^{(i) 1}\right\rangle=-2 q_{1}^{2}\left|\Lambda_{00}^{(i+1)}\right\rangle+2 r \eta_{1}^{+}\left(L_{2}-2 l_{2}^{\prime}\right)\left|\Lambda_{01}^{(i+1) 1}\right\rangle+r\left(T_{1}-2 t_{1}^{\prime}\right)\left|\Lambda_{01}^{(i+1) 0}\right\rangle \\
& \delta\left|\Lambda_{03}^{(i) 1}\right\rangle=2 q_{1}^{2}\left|\Lambda_{01}^{(i+1) 1}\right\rangle .
\end{aligned}
$$

Using (119)-(122), we can see that the dependence on $\mathcal{P}_{2}^{+}$in $\left|\Lambda^{(i) j}\right\rangle$ can be removed with the help of the gauge transformations. To this end, we should first make gauge transformations with $\left|\Lambda_{01}^{(i+1) 0}\right\rangle$ and $\left|\Lambda_{01}^{(i+1) 1}\right\rangle$, removing $\left|\Lambda_{03}^{(i) 0}\right\rangle$ and $\left|\Lambda_{03}^{(i) 1}\right\rangle$, respectively. Then we should make gauge transformation with the parameter $\left|\Lambda_{00}^{(i+1) 0}\right\rangle$, removing $\left|\Lambda_{02}^{(i) 0}\right\rangle$. Finally, we should make gauge transformation with the parameter $\left|\Lambda^{(i+1) 1}{ }_{00}\right\rangle$, removing $\left|\Lambda_{02}^{(i) 1}\right\rangle$. Thus, we have shown that the dependence on $\mathcal{P}_{2}^{+}$can be eliminated from $\left|\Lambda_{0}^{(i) j}\right\rangle$.

This procedure works perfectly well until the terms linear in $p_{1}^{+}$appear in the gauge functions $\left|\Lambda^{(i+1)}\right\rangle$. When these terms are present, some of the gauge parameters remain unused after eliminating the $\mathcal{P}_{2}^{+}$dependence. Due to the presence of $r$-dependent terms in (119), (121), it is obvious that if one should make a gauge transformation with such a parameter the terms depending on the ghost $\mathcal{P}_{2}^{+}$may appear again. Therefore, one should make gauge transformations with parameters being linear in $p_{1}^{+}$or independent of it, before removing the $\mathcal{P}_{2}^{+}$dependence. The first gauge function where such a term appear is $\left|\Lambda^{(1)}\right\rangle$. Let us consider gauge transformation with this gauge function more carefully.

Suppose that the dependence on the ghost $\mathcal{P}_{2}^{+}$in $\left|\Lambda^{(1)}\right\rangle$ has been removed by a gauge transformation. Let us decompose the gauge functions $\left|\Lambda^{(1)}\right\rangle$ and $\left|\Lambda^{(0)}\right\rangle$ as follows:

$$
\begin{aligned}
&\left|\Lambda_{0}^{(1) 0}\right\rangle=-i p_{1}^{+} \mathcal{P}_{1}^{+}|\omega\rangle+\left(p_{1}^{+}\right)^{2}(\ldots) \\
&\left|\Lambda_{0}^{(1) 1}\right\rangle=\left(p_{1}^{+}\right)^{2}(\ldots) \\
&\left|\Lambda_{0}^{(0) 0}\right\rangle= \mathcal{P}_{1}^{+}|\varepsilon\rangle-i p_{1}^{+}\left|\varepsilon_{1}\right\rangle-i \eta_{1}^{+} p_{1}^{+} \mathcal{P}_{1}^{+}\left|\varepsilon_{2}\right\rangle \\
& \quad-i q_{1}^{+} p_{1}^{+} \mathcal{P}_{1}^{+}\left|\varepsilon_{3}\right\rangle-i \eta_{2}^{+} p_{1}^{+} \mathcal{P}_{1}^{+}\left|\varepsilon_{4}\right\rangle+\left(p_{1}^{+}\right)^{2}(\ldots)+\mathcal{P}_{2}^{+}(\ldots), \\
&\left|\Lambda_{0}^{(0) 1}\right\rangle=-i p_{1}^{+} \mathcal{P}_{1}^{+}\left|\varepsilon_{5}\right\rangle+\left(p_{1}^{+}\right)^{2}(\ldots)+\mathcal{P}_{2}^{+}(\ldots) .
\end{aligned}
$$

As has been shown, we have to make a gauge transformation with a parameter linear in $p_{1}^{+}$ (parameters which do not depend on $p_{1}^{+}$are absent from $\left|\Lambda^{(1)}\right\rangle$ ). Therefore, we use $|\omega\rangle$ to make such a gauge transformation. Since

$$
\delta|\varepsilon\rangle=-T_{1}^{+}|\omega\rangle
$$

we use $|\omega\rangle$ to eliminate the dependence on $b_{2}^{+}$and $f^{+}$from $|\varepsilon\rangle$,

$$
b_{2}|\varepsilon\rangle=f|\varepsilon\rangle=0
$$


and then we remove the $\mathcal{P}_{2}^{+}$dependence from $\left|\Lambda^{(0)}\right\rangle$ as described above.

Let us turn to the gauge-fixing of the fields. We decompose the field in ghosts $\mathcal{P}_{1}^{+}, \mathcal{P}_{2}^{+}$by analogy with the gauge functions,

$$
\left|\chi_{0}^{j}\right\rangle=\left|\chi_{00}^{j}\right\rangle+\mathcal{P}_{1}^{+}\left|\chi_{01}^{j}\right\rangle+\mathcal{P}_{2}^{+}\left|\chi_{02}^{j}\right\rangle+\mathcal{P}_{1}^{+} \mathcal{P}_{2}^{+}\left|\chi_{03}^{j}\right\rangle, \quad j=0,1
$$

with each $\left|\chi_{0 m}^{j}\right\rangle$ being an expansion in $p_{1}^{+}$,

$$
\left|\chi_{0 m}^{j}\right\rangle=\sum_{k=0}\left(p_{1}^{+}\right)^{k}\left|\chi_{0 m}^{j k}\right\rangle
$$

By analogy with the gauge-fixing of $\left|\Lambda^{(0)}\right\rangle$, we have to use $\left|\varepsilon_{i}\right\rangle$ and then remove the $\mathcal{P}_{2}^{+}$dependence from the fields $\left|\chi_{0}^{j}\right\rangle$.

Substituting (125), (126) into (109), (110), we find a gauge transformation for the fields independent of the ghost $p_{1}^{+}$,

$$
\begin{aligned}
\delta\left|\chi_{00}^{00}\right\rangle= & L_{1}^{+}|\varepsilon\rangle+T_{1}^{+}\left|\varepsilon_{1}\right\rangle, \\
\delta\left|\chi_{01}^{00}\right\rangle= & \eta_{1}^{+}\left(-T_{1}^{+}\left|\varepsilon_{2}\right\rangle-L_{1}|\varepsilon\rangle-\left|\varepsilon_{5}\right\rangle\right)+\eta_{2}^{+}\left(-T_{1}^{+}\left|\varepsilon_{4}\right\rangle-L_{2}|\varepsilon\rangle-\left|\varepsilon_{3}\right\rangle\right) \\
& +q_{1}^{+}\left(-T_{1}^{+}\left|\varepsilon_{3}\right\rangle-T_{1}|\varepsilon\rangle\right), \\
\delta\left|\chi_{01}^{10}\right\rangle= & -T_{0}|\varepsilon\rangle-2\left|\varepsilon_{1}\right\rangle-T_{1}^{+}\left|\varepsilon_{5}\right\rangle .
\end{aligned}
$$

Thus, we can first remove the $b_{2}^{+}$and $f^{+}$dependence of $\left|\chi_{00}^{00}\right\rangle$, using $\left|\varepsilon_{1}\right\rangle$, and then remove the $b_{1}^{+}$dependence of $\left|\chi_{00}^{00}\right\rangle$, using the restricted (128) gauge parameter $|\varepsilon\rangle$. We then remove the $b_{2}^{+}$ and $f^{+}$dependence of $\left|\chi_{01}^{10}\right\rangle$ and $\left|\chi_{01}^{00}\right\rangle$, using the gauge parameters $\left|\varepsilon_{5}\right\rangle,\left|\varepsilon_{2}\right\rangle,\left|\varepsilon_{3}\right\rangle$, $\left|\varepsilon_{4}\right\rangle$. After this, we remove the $\mathcal{P}_{2}^{+}$dependence of the fields. Now, all the gauge parameters have been used, and we have the following conditions for the fields:

$$
\begin{aligned}
& b_{1}\left|\chi_{00}^{00}\right\rangle=b_{2}\left|\chi_{00}^{00}\right\rangle=f\left|\chi_{00}^{00}\right\rangle=0, \\
& b_{2}\left|\chi_{01}^{j 0}\right\rangle=f\left|\chi_{01}^{j 0}\right\rangle=0, \quad j=0,1, \\
& \eta_{2}\left|\chi_{0}^{0}\right\rangle=\eta_{2}\left|\chi_{0}^{1}\right\rangle=0 .
\end{aligned}
$$

Since $g h\left(\left|\chi_{00}^{00}\right\rangle\right)=0$, it does not depend on ghost "coordinates" and due to (134) we conclude, after gauge-fixing, that

$$
\left|\chi_{00}^{00}\right\rangle=|\Phi\rangle
$$

where $|\Phi\rangle$ is the physical field (15).

Let us turn to removing the auxiliary fields with the help of a part of the equations of motion.

\subsection{Removing auxiliary fields by equations of motion}

Let us decompose equation (105) in $\mathcal{P}_{1}^{+}, \mathcal{P}_{2}^{+}$and consider the coefficients of $\mathcal{P}_{2}^{+}$-dependent parts:

$$
\begin{aligned}
-2 \mathcal{P}_{2}^{+} \mathcal{P}_{1}^{+}: & q_{1}^{2}\left|\chi_{01}^{0}\right\rangle=0, \\
-2 \mathcal{P}_{2}^{+}: & q_{1}^{2}\left|\chi_{00}^{0}\right\rangle-r \eta_{1}^{+}\left(L_{2}-2 l_{2}^{\prime}\right)\left|\chi_{01}^{0}\right\rangle=0 .
\end{aligned}
$$

Using (138), we conclude that $\left|\chi_{01}^{0}\right\rangle$ contains no terms of an order larger than the first order in $p_{1}^{+}$,

$$
\left|\chi_{01}^{0}\right\rangle=\left|\chi_{01}^{00}\right\rangle+p_{1}^{+}\left|\chi_{01}^{01}\right\rangle .
$$


Substituting (140) to (139), we find

$$
\left|\chi_{00}^{0}\right\rangle=\sum_{k=0}^{3}\left(p_{1}^{+}\right)^{k}\left|\chi_{00}^{0 k}\right\rangle
$$

where

$$
\left|\chi_{00}^{02}\right\rangle=-\frac{r}{2} \eta_{1}^{+}\left(L_{2}-2 l_{2}^{\prime}\right)\left|\chi_{01}^{00}\right\rangle, \quad\left|\chi_{00}^{03}\right\rangle=-\frac{r}{6} \eta_{1}^{+}\left(L_{2}-2 l_{2}^{\prime}\right)\left|\chi_{01}^{01}\right\rangle
$$

In a similar way, we decompose equation (106) and obtain

$$
\begin{aligned}
-2 \mathcal{P}_{2}^{+} \mathcal{P}_{1}^{+}: & q_{1}^{2}\left|\chi_{01}^{1}\right\rangle=0 \\
-2 \mathcal{P}_{2}^{+}: & q_{1}^{2}\left|\chi_{00}^{1}\right\rangle-r \eta_{1}^{+}\left(L_{2}-2 l_{2}^{\prime}\right)\left|\chi_{01}^{1}\right\rangle-\frac{r}{2}\left(T_{1}-2 t_{1}^{\prime}\right)\left|\chi_{01}^{0}\right\rangle=0 .
\end{aligned}
$$

These equations have the following solution:9

$$
\left|\chi_{01}^{1}\right\rangle=\left|\chi_{01}^{10}\right\rangle+p_{1}^{+}\left|\chi_{01}^{11}\right\rangle, \quad\left|\chi_{00}^{1}\right\rangle=\sum_{k=1}^{3}\left(p_{1}^{+}\right)^{k}\left|\chi_{00}^{1 k}\right\rangle
$$

where

$$
\begin{aligned}
\left|\chi_{00}^{12}\right\rangle & =-\frac{r}{2} \eta_{1}^{+}\left(L_{2}-2 l_{2}^{\prime}\right)\left|\chi_{01}^{10}\right\rangle-\frac{r}{4}\left(T_{1}-2 t_{1}^{\prime}\right)\left|\chi_{01}^{00}\right\rangle \\
\left|\chi_{00}^{13}\right\rangle & =-\frac{r}{6} \eta_{1}^{+}\left(L_{2}-2 l_{2}^{\prime}\right)\left|\chi_{01}^{11}\right\rangle-\frac{r}{12}\left(T_{1}-2 t_{1}^{\prime}\right)\left|\chi_{01}^{01}\right\rangle .
\end{aligned}
$$

Let us now consider a part of equations (105), (106) containing the physical field $\left|\chi_{00}^{00}\right\rangle=|\Phi\rangle$ (137),

$$
\begin{array}{cc} 
& \left(t_{0}+\tilde{\gamma} m_{0}\right)|\Phi\rangle+i T_{1}^{+}\left|\chi_{00}^{11}\right\rangle+L_{1}^{+}\left|\chi_{01}^{10}\right\rangle-r\left(L_{2}^{+}-2 l_{2}^{++}\right)\left|\chi_{010}^{001}\right\rangle=0 \\
q_{1}^{+}: \quad & t_{1}|\Phi\rangle+i T_{1}^{+}\left|\chi_{000}^{011}\right\rangle+L_{1}^{+}\left|\chi_{010}^{001}\right\rangle-\left|\chi_{01}^{10}\right\rangle=0 \\
\eta_{1}^{+}: \quad & l_{1}|\Phi\rangle-i T_{1}^{+}\left|\chi_{001}^{010}\right\rangle+L_{1}^{+}\left|\chi_{011}^{000}\right\rangle-i\left|\chi_{00}^{11}\right\rangle \\
& -2 r\left(L_{2}^{+}-2 l_{2}^{++}\right)\left|\chi_{012}^{000}\right\rangle+\frac{3 r}{4}\left(T_{1}^{+}-2 t_{1}^{\prime+}\right)\left|\chi_{010}^{001}\right\rangle-T_{0}\left|\chi_{01}^{10}\right\rangle=0 \\
\eta_{2}^{+}: & l_{2}|\Phi\rangle-i T_{1}^{+}\left|\chi_{002}^{010}\right\rangle+i\left|\chi_{000}^{011}\right\rangle+L_{1}^{+}\left|\chi_{012}^{000}\right\rangle-\left|\chi_{011}^{000}\right\rangle=0
\end{array}
$$

where we have decomposed the fields $\left|\chi_{01}^{00}\right\rangle$ and $\left|\chi_{00}^{01}\right\rangle$ as follows:

$$
\left|\chi_{01}^{00}\right\rangle=q_{1}^{+}\left|\chi_{010}^{001}\right\rangle+\eta_{1}^{+}\left|\chi_{011}^{000}\right\rangle+\eta_{2}^{+}\left|\chi_{012}^{000}\right\rangle, \quad\left|\chi_{00}^{01}\right\rangle=q_{1}^{+}\left|\chi_{000}^{011}\right\rangle+\eta_{1}^{+}\left|\chi_{001}^{010}\right\rangle+\eta_{2}^{+}\left|\chi_{002}^{010}\right\rangle .
$$

Acting on (149) by the operators $f$ and $b_{2}$ and taking into account the gauge-fixing condition (134), (135), we obtain, respectively,

$$
f T_{1}^{+}\left|\chi_{000}^{011}\right\rangle=0, \quad b_{2} T_{1}^{+}\left|\chi_{000}^{011}\right\rangle=0
$$

These equations yield

$$
\left|\chi_{000}^{011}\right\rangle=0
$$

\footnotetext{
${ }^{9}$ The term $\left|\chi_{00}^{10}\right\rangle$ corresponding to $\left(p_{1}^{+}\right)^{0}$ is absent in the sum of (145) due to the ghost number restriction (101).
} 
Acting on (151) by $f$ and $b_{2}$, we obtain

$$
f T_{1}^{+}\left|\chi_{002}^{010}\right\rangle=b_{2} T_{1}^{+}\left|\chi_{002}^{010}\right\rangle=0 \Longrightarrow\left|\chi_{002}^{010}\right\rangle=0
$$

We now act on equation (148) by the operator $f$,

$$
f T_{1}^{+}\left|\chi_{00}^{11}\right\rangle=0
$$

and then presenting the vector $\left|\chi_{00}^{11}\right\rangle$ as a power series in $f^{+}$

$$
\left|\chi_{00}^{11}\right\rangle=\left|a_{00}^{11}\right\rangle+f^{+}\left|b_{00}^{11}\right\rangle,
$$

we have

$$
\left|a_{00}^{11}\right\rangle=t_{1}^{+}\left|b_{00}^{11}\right\rangle
$$

Substituting this result into equation (148) and acting twice by the operator $b_{2}$ on the resulting equations, we arrive at

$$
b_{2}\left|b_{00}^{11}\right\rangle=0
$$

Finally, equation (148) acquires the form

$$
\left(t_{0}+\tilde{\gamma} m_{0}\right)|\Phi\rangle+2 i L_{2}^{+}\left|b_{00}^{11}\right\rangle+L_{1}^{+}\left|\chi_{01}^{10}\right\rangle-r\left(L_{2}^{+}-2 l_{2}^{\prime+}\right)\left|\chi_{010}^{001}\right\rangle=0
$$

Let us present the state vectors $\left|b_{00}^{11}\right\rangle,\left|\chi_{01}^{10}\right\rangle,\left|\chi_{010}^{001}\right\rangle$ as a power series in $b_{1}^{+}$,

$$
\left|b_{00}^{11}\right\rangle=\sum_{k=0}^{n-2}\left(b_{1}^{+}\right)^{k}\left|b_{00}^{11}\right\rangle^{k}, \quad\left|\chi_{01}^{10}\right\rangle=\sum_{k=0}^{n-1}\left(b_{1}^{+}\right)^{k}\left|\chi_{01}^{10}\right\rangle^{k}, \quad\left|\chi_{010}^{001}\right\rangle=\sum_{k=0}^{n-2}\left(b_{1}^{+}\right)^{k}\left|\chi_{010}^{001}\right\rangle^{k}
$$

and then, presenting equations (160) and (149) as power series in $b_{1}^{+}$and $b_{2}^{+}$, we have

$$
\begin{aligned}
& \left(b_{1}^{+}\right)^{n}: \quad m_{1}\left|\chi_{01}^{10}\right\rangle^{n-1}=0, \\
& \left(b_{1}^{+}\right)^{n-1}: \quad m_{1}\left|\chi_{01}^{10}\right\rangle^{n-2}=-l_{1}^{+}\left|\chi_{01}^{10}\right\rangle^{n-1}, \\
& 1 \leq k \leq n-2 \quad\left(b_{1}^{+}\right)^{k}: \quad m_{1}\left|\chi_{01}^{10}\right\rangle^{k-1}=-l_{1}^{+}\left|\chi_{01}^{10}\right\rangle^{k}-2 i l_{2}^{+}\left|b_{00}^{11}\right\rangle^{k}+r l_{2}^{+}\left|\chi_{010}^{001}\right\rangle^{k} \text {, } \\
& \left(b_{1}^{+}\right)^{0}: \quad\left(t_{0}+\tilde{\gamma} m_{0}\right)|\Phi\rangle=-2 i l_{2}^{+}\left|b_{00}^{11}\right\rangle^{0}-l_{1}^{+}\left|\chi_{01}^{10}\right\rangle^{0}+r l_{2}^{+}\left|\chi_{010}^{001}\right\rangle^{0}, \\
& 0 \leq k \leq n-2 \quad b_{2}^{+}\left(b_{1}^{+}\right)^{k}: \quad\left|b_{00}^{11}\right\rangle^{k}=\frac{i r}{2}\left|\chi_{010}^{001}\right\rangle^{k} \\
& \left(b_{1}^{+}\right)^{n-1}: \quad m_{1}\left|\chi_{010}^{001}\right\rangle^{n-2}=\left|\chi_{01}^{10}\right\rangle^{n-1}, \\
& 1 \leq k \leq n-2 \quad\left(b_{1}^{+}\right)^{k}: \quad m_{1}\left|\chi_{010}^{001}\right\rangle^{k-1}=\left|\chi_{01}^{10}\right\rangle^{k}-l_{1}^{+}\left|\chi_{010}^{001}\right\rangle^{k} \\
& \left(b_{1}^{+}\right)^{0}: \quad t_{1}|\Phi\rangle=\left|\chi_{01}^{10}\right\rangle^{0}-l_{1}^{+}\left|\chi_{010}^{001}\right\rangle^{0} \text {. }
\end{aligned}
$$

Using (162) and (163), we have $\left|\chi_{01}^{10}\right\rangle^{n-1}=\left|\chi_{01}^{10}\right\rangle^{n-2}=0$. Substituting this result into (167) and then into (168) for $k=n-2$, we obtain $\left|\chi_{010}^{001}\right\rangle^{n-2}=\left|\chi_{010}^{001}\right\rangle^{n-3}=0$. Turning to equation (166) for $k=n-2$ and $k=n-3$, we conclude that $\left|b_{00}^{11}\right\rangle^{n-2}=\left|b_{00}^{11}\right\rangle^{n-3}=0$. We now repeat the procedure starting from equation (164) for $k=n-2$ and $k=n-3$. Finally, we obtain

$$
\begin{aligned}
& \left(t_{0}+\tilde{\gamma} m_{0}\right)|\Phi\rangle=0, \quad t_{1}|\Phi\rangle=0, \\
& \left|\chi_{01}^{10}\right\rangle=\left|\chi_{010}^{001}\right\rangle=0, \quad\left|b_{00}^{11}\right\rangle=0 \quad \Longrightarrow\left|\chi_{00}^{11}\right\rangle=0 .
\end{aligned}
$$


Using (170), we can see that the physical state (5) satisfies (1), (22), or equivalently (45), provided that condition (115) is taken into account.

Let us now turn to equations (150), (151). Taking into account (154), (155), (171), we can see that these two equations read

$$
\begin{aligned}
& l_{1}|\Phi\rangle-i T_{1}^{+}\left|\chi_{001}^{010}\right\rangle+L_{1}^{+}\left|\chi_{011}^{000}\right\rangle-2 r\left(L_{2}^{+}-2 l_{2}^{\prime+}\right)\left|\chi_{012}^{000}\right\rangle=0 \\
& l_{2}|\Phi\rangle+L_{1}^{+}\left|\chi_{012}^{000}\right\rangle-\left|\chi_{011}^{000}\right\rangle=0
\end{aligned}
$$

They are analogous to (148) and (149), where $\left|\chi_{000}^{011}\right\rangle=0$ in (154) is taken into account. Therefore, we can repeat the procedure that has been carried out with equations (148), (149), and thus we arrive at the conclusion that

$$
\begin{aligned}
& l_{1}|\Phi\rangle=l_{2}|\Phi\rangle=0 \\
& \left|\chi_{001}^{010}\right\rangle=\left|\chi_{012}^{000}\right\rangle=\left|\chi_{011}^{000}\right\rangle=0
\end{aligned}
$$

Equations (174) are consequences of (170); they do not impose any additional restrictions on the physical state (5). Collecting (154), (155), (171), (175) and (152), we have

$$
\left|\chi_{01}^{10}\right\rangle=\left|\chi_{00}^{11}\right\rangle=\left|\chi_{01}^{00}\right\rangle=\left|\chi_{00}^{01}\right\rangle=0
$$

Observing (140), (141), (142) and (145), (146), (147), we can see that it remains to show that $\left|\chi_{01}^{01}\right\rangle=\left|\chi_{01}^{11}\right\rangle=0$. To prove this fact, we decompose (105) and (106) in the ghosts $p_{1}^{+}, \mathcal{P}_{1}^{+}$and consider the equations which are the coefficients at $\left(p_{1}^{+}\right)^{0} \mathcal{P}_{1}^{+}$. With allowance for (176)), these equations read

$$
i T_{1}^{+}\left|\chi_{01}^{01}\right\rangle-\eta_{2}^{+} p_{1}\left|\chi_{01}^{01}\right\rangle=0, \quad i T_{1}^{+}\left|\chi_{01}^{11}\right\rangle-\eta_{2}^{+} p_{1}\left|\chi_{01}^{11}\right\rangle=0
$$

respectively. Decomposing $\left|\chi_{01}^{01}\right\rangle,\left|\chi_{01}^{11}\right\rangle$ in ghost "coordinates",

$$
\left|\chi_{01}^{01}\right\rangle=q_{1}^{+}\left|\chi_{010}^{011}\right\rangle+\eta_{1}^{+}\left|\chi_{011}^{010}\right\rangle+\eta_{2}^{+}\left|\chi_{012}^{010}\right\rangle, \quad\left|\chi_{01}^{11}\right\rangle=q_{1}^{+}\left|\chi_{010}^{111}\right\rangle+\eta_{1}^{+}\left|\chi_{011}^{110}\right\rangle+\eta_{2}^{+}\left|\chi_{012}^{110}\right\rangle
$$

and substituting the result into (177), we obtain equations for the coefficients of (178) in the form

$$
T_{1}^{+}\left|\chi_{01 n}^{j 1 i}\right\rangle=0 \quad \Longrightarrow \quad\left|\chi_{01 n}^{j 1 i}\right\rangle=0
$$

Therefore, all the coefficient in decompositions (178) of $\left|\chi_{01}^{01}\right\rangle,\left|\chi_{01}^{11}\right\rangle$ are equal to zero, and we conclude that

$$
\left|\chi_{01}^{01}\right\rangle=\left|\chi_{01}^{11}\right\rangle=0
$$

Thus, we have shown that after the gauge fixing (134)-(136) all the auxiliary fields become equal to zero, (176), (180), and the physical state $|\Phi\rangle=\left|\chi_{00}^{00}\right\rangle$ (137) obeys equations (1), (2).

Let us now consider some examples of the Lagrangian construction procedure.

\section{$7 \quad$ Examples}

Here, we shall illustrate the general procedure of gauge-invariant Lagrangian construction by using the examples of fermionic fields of spin $1 / 2$ and $3 / 2$. 


\section{$7.1 \quad$ Spin-1/2 field}

For a fermionic field of spin $s=\frac{1}{2}$, we have $h=2-\frac{d}{2}$. Then the only nonvanishing vector $\left|\chi_{0}^{0}\right\rangle_{0}$ subject to condition (107) and having the proper ghost number (92) has the form

$$
\left|\chi_{0}^{0}\right\rangle_{0}=\psi(x)|0\rangle, \quad{ }_{0}\left\langle\tilde{\chi}_{0}^{0}\right|={ }_{0}\langle 0| \psi^{+}(x) \tilde{\gamma}^{0} .
$$

Then, due to (75), (86) for $K_{0}=|0\rangle\langle 0|+\ldots$, (115), the action implied by (108) has the form

$$
\mathcal{S}_{0}={ }_{0}\left\langle\tilde{\chi}_{0}^{0}\left|K_{0} T_{0}\right| \chi_{0}^{0}\right\rangle_{0}=-\int d^{d} x \sqrt{|g|} \bar{\psi}\left\{i \gamma^{\mu} \nabla_{\mu}-m-r^{\frac{1}{2}}\left(\frac{d}{2}-2\right)\right\} \psi
$$

Here, we have applied the definition (14) for the conventional gamma-matrices and have introduced the Dirac-conjugate spinor $\bar{\psi}, \bar{\psi}=\psi^{+} \gamma^{0}$. Thus, we can see that the action (182) reproduces equation (1) for $n=0$, which corresponds to spin- $1 / 2$ field.

\section{$7.2 \quad$ Spin-3/2 field}

In the case of a spin-3/2 field, we have $n=1, h=1-d / 2, m_{0}=m+r^{\frac{1}{2}}(d-2) / 2$. Since $i_{\max }=0$, the corresponding Lagrangian formulation is an irreducible gauge theory. Due to $g h\left(\left|\Lambda_{0}^{1}\right\rangle_{1}\right)=$ -2), the nonvanishing fields $\left|\chi_{0}^{0}\right\rangle_{1},\left|\chi_{0}^{1}\right\rangle_{1}$ and the gauge parameter $\left|\Lambda_{0}^{0}\right\rangle_{1}$, for $\left|\Lambda_{0}^{1}\right\rangle_{1} \equiv 0$, possess the following Grassmann grading and ghost number distributions:

$$
(\varepsilon, g h)\left(\left|\chi_{0}^{0}\right\rangle_{1}\right)=(1,0), \quad(\varepsilon, g h)\left(\left|\chi_{0}^{1}\right\rangle_{1}\right)=(1,-1), \quad(\varepsilon, g h)\left(\left|\Lambda_{0}^{0}\right\rangle_{1}\right)=(0,-1) .
$$

These conditions determine the dependence of the fields and gauge parameter on the oscillator variables in a unique form:

$$
\begin{array}{ll}
\left|\chi_{0}^{0}\right\rangle_{1}=\left[-i a^{+\mu} \psi_{\mu}(x)+f^{+} \tilde{\gamma} \psi(x)+b_{1}^{+} \varphi(x)\right]|0\rangle, & \left|\chi_{0}^{1}\right\rangle_{1}=\left[\mathcal{P}_{1}^{+} \tilde{\gamma} \chi(x)+i p_{1}^{+} \chi_{1}(x)\right]|0\rangle, \\
{ }_{1}\left\langle\tilde{\chi}_{0}^{0}\right|=\langle 0|\left[i \psi_{\mu}^{+}(x) a^{\mu}+\psi^{+}(x) \tilde{\gamma} f+\varphi^{+}(x) b_{1}\right] \tilde{\gamma}^{0}, & { }_{1}\left\langle\tilde{\chi}_{0}^{1}\right|=\langle 0|\left[\chi^{+}(x) \tilde{\gamma} \mathcal{P}_{1}-\chi_{1}^{+} i p_{1}\right] \tilde{\gamma}^{0}, \\
\left|\Lambda_{0}^{0}\right\rangle_{1}=\left[\mathcal{P}_{1}^{+} \xi_{1}(x)-i p_{1}^{+} \tilde{\gamma} \xi_{2}(x)\right]|0\rangle . &
\end{array}
$$

Substituting (184), (185) into (108), we find the action (up to an overall factor) for a spin-3/2 field interacting with the AdS background:

$$
\begin{aligned}
\mathcal{S}_{1}= & \int d^{d} x \sqrt{|g|}\left[\bar{\psi}^{\mu}\left\{\left[i \gamma^{\nu} \nabla_{\nu}-m_{0}\right] \psi_{\mu}-\nabla_{\mu} \chi+i \gamma_{\mu} \chi_{1}\right\}\right. \\
& +\left[(d-2) \bar{\psi}-\frac{m_{0}}{m_{1}} \bar{\varphi}\right]\left\{\left[i \gamma^{\mu} \nabla_{\mu}+m_{0}\right] \psi-\frac{r(d-1)}{2 m_{1}} \varphi-\chi_{1}\right\} \\
& -\left[\frac{M^{2}}{m_{1}^{2}} \bar{\varphi}+\frac{m_{0}}{m_{1}} \bar{\psi}\right]\left\{\left[i \gamma^{\sigma} \nabla_{\sigma}-m_{0}\right] \varphi-2 m_{1} \psi-m_{1} \chi\right\} \\
& +\bar{\chi}\left\{\left[i \gamma^{\mu} \nabla_{\mu}+m_{0}\right] \chi+\chi_{1}+\nabla^{\mu} \psi_{\mu}+m_{0} \psi+\frac{M^{2}}{m_{1}} \varphi\right\} \\
& \left.-\bar{\chi}_{1}\left\{i \gamma^{\mu} \psi_{\mu}+(d-2) \psi-\chi-\frac{m_{0}}{m_{1}} \varphi\right\}\right]
\end{aligned}
$$

where $M^{2}=m_{0}^{2}-\frac{1}{4} r(d-1)(d-2)$. To obtain the action (187), we have used the expressions for the operators $K_{1}(\mathbf{7 5})$, (86). Substituting (184)-(186) into (109), (110), we find the gauge transformations

$$
\begin{aligned}
& \delta \psi_{\mu}=\nabla_{\mu} \xi_{1}+i \gamma_{\mu} \xi_{2}, \quad \delta \psi=\xi_{2}, \quad \delta \varphi=m_{1} \xi_{1} \\
& \delta \chi=\left[i \gamma^{\mu} \nabla_{\mu}-m_{0}\right] \xi_{1}-2 \xi_{2}, \quad \delta \chi_{1}=\left[i \gamma^{\mu} \nabla_{\mu}+m_{0}\right] \xi_{2}-\frac{r}{2}(d-1) \xi_{1} \text {. }
\end{aligned}
$$


Let us present the action in terms of one physical field $\psi_{\mu}$. To this end, we get rid of the fields $\varphi, \psi$, by using their gauge transformations and the gauge parameters $\xi_{1}, \xi_{2}$, respectively. Having expressed the field $\chi$, using the equation of motion $\chi=i \gamma^{\mu} \psi_{\mu}$, we can see that the terms with the Lagrangian multiplier $\chi_{1}$ turn to zero. As a result, we obtain

$$
\mathcal{L}_{R S}=\bar{\psi}^{\mu}\left(i \gamma^{\sigma} \nabla_{\sigma}-m_{0}\right) \psi_{\mu}-i \bar{\psi}^{\mu}\left(\gamma_{\nu} \nabla_{\mu}+\gamma_{\mu} \nabla_{\nu}\right) \psi^{\nu}+\bar{\psi}^{\nu} \gamma_{\nu}\left(i \gamma^{\sigma} \nabla_{\sigma}+m_{0}\right) \gamma^{\mu} \psi_{\mu} .
$$

This is a generalization of the Rarita-Schwinger Lagrangian to a $d$-dimensional AdS space.

\section{Conclusion}

We have constructed a gauge-invariant Lagrangian formulation of half-integer totally symmetric higher spin fields in the AdS space of any dimension in the "metric-like" formulation. The results of this study are most general and apply to both massive and massless fermionic higher spin fields in the AdS, Minkowski, and dS10 spaces.

Starting from embedding the fermionic higher spin fields into vectors of an auxiliary Fock space, we treat the fields as components of these Fock-space vectors, and, as a result, we reformulate the theory in terms of such vectors. We realize the conditions that define an irreducible representation of the AdS group with a given mass and spin in terms of differential operators acting in this Fock space. The mentioned conditions are interpreted as constraints imposed on the Fock space vectors and generate a closed higher spin nonlinear symmetry superalgebra being the basic object of this study.

It is shown that the derivation of a correct Lagrangian formulation requires a transition to another constraint basis for the original symmetry algebra, which is algebraically equivalent to the initial basis and does not contain the constraints that define an irreducible representation of the AdS group. This set of constraints provides an additive extension of the initial algebra to another operator algebra. Then, as shown in [33], it is necessary to deform the initial algebra. As a result, one obtains a nonlinear superalgebra of enlarged constraints, whose construction by means of the additional operators realizes a special conversion of the initial system of firstand second-class operator constraints into a system of first-class ones with a preservation of the initial algebraic structure for the deformed constraints (see [37] for the elaboration of conversion methods). Due to the nonlinearity of the underlying algebra of the enlarged constraints, the corresponding BRST operator is defined ambiguously, and we construct it in an exact form with a non-vanishing term of third order in powers of ghosts for the case of a supersymmetric ordering of the constraints in commutator relations. It is shown that the resulting BRST operator yields a consistent Lagrangian dynamics for fermionic fields of any spin. The corresponding Lagrangian formulation is constructed in a concise form in terms of a Fock space and proves to be a reducible gauge theory with a finite number of reducibility stages, the number growing with the spin value. It is interesting to observe that the methods developed for the quantization of gauge theories turn out to be extremely efficient for deriving classical gauge-invariant Lagrangians for higher spin field theories, thus reflecting one more side of the BV-BFV duality concept [38], which permits one to construct, by means of a Hamiltonian BFV-BRST charge, the objects used in Lagrangian formalism.

We have proved that the Lagrangian equations of motion (105), (106), after a partial gaugefixing, reproduce the equations corresponding to the relations determining the irreducible representation of the AdS group. This proof completes the derivation of a correct gauge-invariant

\footnotetext{
${ }^{10}$ In the case of HS fields on dS space the corresponding Lagrangians lose the property of reality due to $r^{\frac{1}{2}}$.
} 
Lagrangian formulation for higher spin fermionic fields in the AdS space. As examples demonstrating the general scheme of Lagrangian construction, we have obtained gauge-invariant Lagrangian descriptions of fields with spin- $1 / 2$ and spin- $3 / 2$ in an explicit form. In principle, the constructed description permits one to obtain explicit Lagrangians for any other half-integerspin fields.

The basic results of the present work are given by relations (108), where the Lagrangian action for a field with an arbitrary half-integer spin is constructed, and by (109)-(112), where the gauge transformations for the fields are presented as well as the sequence of reducible gauge transformations for the gauge parameters.

Concluding, we shall discuss two points. First, the gauge-invariant formulation for massive higher spin field theories in the AdS space fields can have very interesting applications in the calculation of a quantum effective action for higher spin massive fields in the AdS space. The Lagrangians of all such models have a gauge-invariant kinetic term and a mass term violating the gauge invariance. Such a structure of a Lagrangian leads to some problems of quantum calculations in a curved space-time. To avoid these problems, it is natural to construct a gauge-invariant formulation by using appropriate Stückelberg fields. Then, one can impose gauge-fixing conditions removing the gauge degeneration of the kinetic term in the Lagrangian of the basic fields and apply the standard techniques for the calculation of an effective action (see the examples of such calculations in [39]). We emphasize that the BRST approach leads automatically to a gauge-invariant Lagrangian with the entire set of appropriate Stückelberg fields. As a result, one has a basis for constructing the effective action of massive higher spin fields in the AdS space. Second, the BRST approach can provide a systematic method of constructing interaction vertices for higher spin fields in the AdS space [30]. Therefore, one can hope that the BRST construction developed in this paper will be useful in deriving interaction vertices for massive higher spin fermionic fields in the AdS space.

\section{Acknowledgements}

A.A.R. is grateful to M. Grigoriev and P. Moshin for useful discussions. The work of I.L.B and V.A.K was partially supported by the INTAS grant, project INTAS-05-7928, the RFBR grant, project No. 06-02-16346, and the grant for LRSS, project No. 4489.2006.2. The work of I.L.B was supported in part by the DFG grant, project No. 436 RUS 113/669/0-3, and the joint RFBR-DFG grant, project No. 06-02-04012. The work of V.A.K was partially supported by the joint DAAD-Mikhail Lomonosov Programme (Referat 325, Kennziffer A/06/16774).

\section{References}

[1] M. Vasiliev, Higher Spin Gauge Theories in Various Dimensions, Fortsch.Phys. 52 (2004) 702-717, arXiv:hep-th/0401177]; D. Sorokin, Introduction to the Classical Theory of Higher Spins, AIP Conf.Proc. 767 (2005) 172-202, arXiv:hep-th/0405069; N. Bouatta, G. Compère, A. Sagnotti, An Introduction to Free Higher-Spin Fields, arXiv:hep-th/0409068]; A. Sagnotti, E. Sezgin, P. Sundell, On higher spin with a strong $S p(2)$ conditions, |arXiv:hep-th/0501156]; X. Bekaert, S. Cnockaert, C. Iazeolla, M.A. Vasiliev, Nonlinear higher spin theories in various dimensions, arXiv:hep-th/0503128].

[2] I.L. Buchbinder, V.A. Krykhtin, V.D. Pershin, On Consistent Equations for Massive Spin-2 Field Coupled to Gravity in String Theory, Phys.Lett. B466 (1999) 216-226, arXiv:hep-th/9908028; I.L. Buchbinder, D.M. Gitman, V.A. Krykhtin, V.D. Pershin, 
Equations of Motion for Massive Spin 2 Field Coupled to Gravity, Nucl.Phys. B584 (2000) 615-640, arXiv:hep-th/9910188] I.L. Buchbinder, D.M. Gitman, V.D. Pershin, Causality of Massive Spin 2 Field in External Gravity, Phys.Lett. B492 (2000) 161-170, arXiv:hep-th/0006144].

[3] S. Deser, A. Waldron, Gauge Invariances and Phases of Massive Higher Spins in (A)dS, Phys. Rev. Lett. 87 (2001) 031601, arXiv:hep-th/0102166; S. Deser, A. Waldron, Partial Masslessness of Higher Spins in (A)dS, Nucl. Phys. B 607 (2001) 577-604, arXiv:hep-th/0103198; Null Propagation of Partially Massless Higher Spins in (A)dS and Cosmological Constant Speculations, Phys. Lett. B513 (2001) 137141, arXiv:hep-th/0105181; K. Hallowell, A. Waldron, Constant Curvature Algebras and Higher Spin Action Generating Functions, Nucl.Phys. B724 (2005) 453-486, arXiv:hep-th/0505255; E.D. Skvortsov, M.A. Vasiliev, Geometric Formulation for Partially Massless Fields, Nucl.Phys. B756 (2006) 117-147, arXiv:hep-th/0601095].

[4] R.R. Metsaev, Massive totally symmetric fields in AdS(d), Phys.Lett. B590 (2004) 95104, arXiv:hep-th/0312297]; R.R. Metsaev, Mixed-symmetry massive fields in AdS(5), Class.Quant.Grav. 22 (2005) 2777-2796, arXiv:hep-th/0412311]; Cubic interaction vertices of massive and massless higher spin fields, Nucl.Phys. B759 (2006) 147-201, arXiv:hep-th/0512342].

[5] S.M. Klishevich, Massive fields with arbitrary integer spin in symmetrical Einstein space, Class.Quant.Grav. 16 (1999) 2915-2927, arXiv:hep-th/9812005]; S.M. Klishevich, On electromagnetic interaction of massive spin-2 particle, Phys.Atom.Nucl. 61 (1998) 1527-1537, arXiv:hep-th/9708150; Massive fields with arbitray half-integer spin in constant electromagnetic field, Int.J.Mod.Phys. A15 (2000) 609-624, arXiv:hep-th/9811030]; Massive fields with arbitray integer spin in homogeneous electromagnetic field, Int.J.Mod.Phys. A15 (2000) 535, [arXiv:hep-th/9810228]; Interaction of massive integer-spin fields, arXiv:hep-th/0002024].

[6] Yu. M. Zinoviev, On Massive High Spin Particles in (A)dS, arXiv:hep-th/0108192; Yu. M. Zinoviev, On Massive Mixed Symmetry Tensor Fields in Minkowski Space and (A)dS, arXiv:hep-th/0211233]; Yu.M. Zinoviev, Massive Spin-2 Supermultiplets, arXiv:hep-th/0206209]; Massive supermultiplets with spin 3/2, arXiv:hep-th/0703118; Massive $\mathrm{N}=1$ supermultiplets with arbitrary superspins, [arXiv:hep-th/0704.1535].

[7] N. Beisert, M. Bianchi, J.F. Morales, H. Samtleben, Higher spin symmetries and $\mathcal{N}=4$ SYM, JHEP 0407 (2004) 058, arXiv:hep-th/0405057]; A.C. Petkou, Holography, duality and higher spin fields, arXiv:hep-th/0410116]; M. Bianchi, P.J. Heslop, F. Riccioni, More on La Grande Bouffe: towards higher spin symmetry breaking in AdS, JHEP 0508 (2005) 088, arXiv:hep-th/0504156; P.J. Heslop, F. Riccioni, On the fermionic Grande Bouffe: more on higher spin symmetry breaking in AdS/CFT, JHEP 0510 (2005) 060, arXiv:hep-th/0508086]; M. Bianchi, V. Didenko, Massive higher spin multiplets and holography, arXiv:hep-th/0502220.

[8] I.L. Buchbinder, S.J. Gates, W.D. Linch, J. Phillips, New 4d, N=1 superfiled theory: model of free massive superspin-3/2 multiplet, Phys.Lett. B535 (2002) 280-288, arXiv:hep-th/0108200; I.L. Buchbinder, S.J. Gates, W.D. Linch, J. Phillips, Dynamical superfiled theory of free massive superspin-1 multiplet, Phys.Lett. B549 (2002) 229236, arXiv:hep-th/0207243]; I.L. Buchbinder, S.J. Gates, S.M. Kuzenko, J. Phillips, Massive 4D, N=1 superspin 1 and 3/2 multiplets and their dualities, JHEP 0502 (2005) 056, 
arXiv:hep-th/0501199]; S. Fedoruk, J. Lukiersky, Massive relativistic models with bosonic counterpart of supersymmetry, Phys.Lett. B632 (2006) 371-378, arXiv:hep-th/0506086.

[9] P. de Medeiros, Massive gauge-invariant field theories on space of constant curvature, Class. Quant. Grav. 21 (2004) 2571-2593, arXiv:hep-th/0311254.

[10] L.Brink, R.R.Metsaev, M.A.Vasiliev, How massless are massless fields in $A d S_{d}$, Nucl. Phys. B586 (2000) 183-205, arXiv:hep-th/0005136]; K.B. Alkalaev, M.A. Vasiliev, N=1 Supersymmetric Theory of Higher Spin Gauge Fields in $A d S(5)$ at the Cubic Level, Nucl.Phys. B655 (2003) 57-92, arXiv:hep-th/0206068]; K.B. Alkalaev, Two-column higher spin massless fields in $A d S(d)$, Theor.Math.Phys. 140 (2004) 1253-1263, arXiv:hep-th/0311212; O.V. Shaynkman, I.Yu. Tipunin, M.A. Vasiliev, Unfolded form of conformal equations in $M$ dimensions and $o(M+2)$-modules, Rev.Math.Phys. 18 (2006) 823-886, arXiv:hep-th/0401086]; K.B. Alkalaev, O.V. Shaynkman, M.A. Vasiliev, On the frame - like formulation of mixed symmetry massless fields in (A)dS(d), Nucl.Phys.B 692 (2004) 363-393 [arXiv:hep-th/0311164]; Lagrangian formulation for free mixed-symmetry bosonic gauge fields in (A)dS(d), JHEP 0508, (2005) 069, arXiv:hep-th/0501108]; K.B. Alkalaev, O.V. Shaynkman, M.A. Vasiliev, Frame-like formulation for free mixed-symmetry bosonic massless higher spin fields in AdS(d), arXiv:hep-th/0601225].

[11] R.R.Metsaev, Free totally (anti)symmetric massless fermionic fields in d-dimensional anti-de Sitter space, Class.Quant.Grav. 14 (1997) L115-L121, arXiv:hep-th/9707066; Fermionic fields in the d-dimensional anti-de Sitter spacetime, Phys.Lett. B419 (1998) 49-56, arXiv:hep-th/9802097]; Arbitrary spin massless bosonic fields in d-dimensional anti-de Sitter space, |arXiv:hep-th/9810231]; Light-cone form of field dynamics in antide Sitter space-time and AdS/CFT correspondence, Nucl. Phys. B563 (1999) 295-348, arXiv:hep-th/9906217]; Massless arbitrary spin fields in AdS(5) Phys. Lett. B531 (2002) 152-160, [arXiv:hep-th/0201226].

[12] R.R. Metsaev, Massless mixed symmetry bosonic free fields in d-dimensional anti-de Sitter space-time, Phys. Lett. B354 (1995) 78-84; Fermionic fields in the d-dimensional anti-de Sitter spacetime, Phys. Lett. B419 (1998) 49-56, [arXiv:hep-th/9802097].

[13] D. Francia, A. Sagnotti, Free geometric equations for higher spins, Phys. Lett. B543 (2002) 303-310, arXiv:hep-th/0207002]; On the geometry of higher spin gauge fields, Class. Quant. Grav. 20 (2003) S473-S486, arXiv:hep-th/0212185]; Minimal Local Lagrangians for Higher-Spin Geometry, Phys. Lett. B624 (2005) 93-104, arXiv:hep-th/0507144; A. Sagnotti, M. Tsulaia, On higher spins and the tensionless limit of String Theory, Nucl. Phys. B682 (2004) 83-116, arXiv:hep-th/0311257); A. Fotopoulos, K.L. Panigrahi, M. Tsulaia, Lagrangian formulation of Higher Spin Theories on AdS, Phys.Rev. D74 (2006) 085029, arXiv:hep-th/0607248]; D. Francia, J. Mourad, A. Sagnotti, Current exchanges and unconstrained higher spins, [arXiv; hep-th/0701163]; I.L. Buchbinder, A.V. Galajinsky, V.A. Krykhtin, Quartet unconstrained formulation for massless higher spin fields, [arxiv: hep-th/0702161].

[14] F. Kristiansson, P. Rajan, Scalar Field Corrections to $\mathrm{AdS}_{4}$ Gravity from Higher Spin Gauge Theory, JHEP 0304 (2003) 009, arXiv:hep-th/0303202].

[15] X. Bekaert, N. Boulaunger, S. Cnockraert, Spin three gauge filed theory revised, JHEP 0601 (2006) 052, arXiv:hep-th/0508048. 
[16] G. Bonelli, On the Tensionless Limit of Bosonic Strings, Infinite Symmetries and Higher Spins, Nucl. Phys. B669 (2003) 159-172, arXiv:hep-th/0305155; G. Barnich, G. Bonelli, M. Grigoriev, From BRST to light-cone description of higher spin gauge fields, arXiv:hep-th/0502232].

[17] M. Plyushchay, D. Sorokin, M. Tsulaia, Higher Spins from Tensorial Charges and $O S p(N \mid 2 n)$ Symmetry, JHEP 0304 (2003) 013, arXiv:hep-th/0301067]; GL Flatness of $O S p(1 \mid 2 n)$ and Higher Spin Field Theory from Dynamics in Tensorial Space, arXiv:hep-th/0310297]; I. Bandos, X. Bekaert, J.A. Azcarraga, D. Sorokin, M. Tsulaia, Dynamics of higher spin fields and tensorial space, JHEP 0505 (2005) 031, arXiv:hep-th/0501113; E. Ivanov, J. Lukiersky, Higher spins from nonliner realizations of $O S p(1 \mid 8)$, Phys.Lett. B624 (2005) 304-315, arXiv:hep-th/0505216]; S. Fedoruk, E. Ivanov, Master higher spin particle, Class.Quant.Grav. 23 (2006) 5195-5214, arXiv:hep-th/0604111]; S. Fedoruk, E. Ivanov, J. Lukiersky, Massless higher spin D=4 superparticle with both $\mathcal{N}=1$ supersymmetry and its bosonic counterpart, Phys.Lett. B641 (2006) 226-236, arXiv:hep-th/0606053.

[18] G. Barnich, M. Grigoriev, A. Semikhatov, I. Tipunin, Parent field theory and unfolding in BRST first-quantized terms, Commun. Math. Phys. 260 (2005) 147-181, arXiv:hep-th/0406192]; G. Barnich, M. Grigoriev, Parent form for higher spin fields on anti-de Sitter space, |arXiv:hep-th/0602166|; M. Grigoriev, Off-shell Gauge Fields from BRST Quantization, arXiv:hep-th/0605089.

[19] C. Fronsdal, Singletons and massless, integer-spin fileds on de Sitter space, Phys. Rev. D20 (1979) 848-856; J. Fang, C. Fronsdal, Massless, half-integer-spin fields in de Sitter space, Phys. Rev. D22 (1980) 1361-1367; M.A. Vasiliev, Free massless fermionic fields of arbitrary spin in D-dimensional anti-de Sitter space, Nucl. Phys. B301 (1988) 26-51; V.E. Lopatin, M.A. Vasiliev, Free massless bosonic fields of arbitrary spin in D-dimensional de Sitter space, Mod. Phys. Lett. A3 (1998) 257-265.

[20] E. S. Fradkin, M. A. Vasiliev, Cubic Interaction In Extended Theories Of Massless Higher Spin Fields, Nucl. Phys. B 291 (1987) 141; Candidate To The Role Of Higher Spin Symmetry, Annals Phys. 177 (1987) 63; On The Gravitational Interaction Of Massless Higher Spin Fields, Phys. Lett. B 189 (1987) 89-95; M. A. Vasiliev, Consistent equation for interacting gauge fields of all spins in (3+1)-dimensions, Phys. Lett. B 243 (1990) 378-382; Cubic interactions of bosonic higher spin gauge fields in AdS(5), Nucl. Phys. B 616 (2001) 106-162 [Erratum-ibid. B 652 (2003) 407] [arXiv:hep-th/0106200]; Algebraic aspects of the higher spin problem, Phys. Lett. B 257 (1991) 111-118; More on equations of motion for interacting massless fields of all spins in (3+1)-dimensions, Phys. Lett. B 285 (1992) 225-234; Class. Quant. Grav. 8, (1991) 1387; Nonlinear equations for symmetric massless higher spin fields in (A)dS(d), Phys. Lett. B 567 (2003) 139-151, arXiv:hep-th/0304049.

[21] E. Sezgin, P. Sundell, Doubletons and 5-D higher spin gauge theory, JHEP 0109 (2001) 036 arXiv:hep-th/0105001); Towards massless higher spin extension of $\mathrm{D}=5, \mathrm{~N}=8$ gauged supergravity, JHEP 0109 (2001) 025, |arXiv:hep-th/0107186|; Analysis of higher spin field equations in four-dimensions, JHEP 0207 (2002) 055, |arXiv:hep-th/0205132]; Holography in 4D (Super) Higher Spin Theories and a Test via Cubic Scalar Couplings, JHEP 0507 (2005) 044, arXiv:hep-th/0305040]; An Exact Solution of 4D Higher-Spin Gauge Theory, Nucl.Phys. B762 (2007) 1-37, |arXiv:hep-th/0508158]; J. Engquist, E. Sezgin, P. Sundell, On $\mathrm{N}=1, \mathrm{~N}=2, \mathrm{~N}=4$ higher spin gauge theories in four-dimensions, Class. Quant. Grav. 19 (2002) 6175-6196, arXiv:hep-th/0207101. 
[22] J.M. Maldacena, The large N limit of superconformal field theories and supergravity, Adv.Theor.math.Phys. 2 (1998) 231, arXiv:hep-th/9711200.

[23] M. Fierz, W. Pauli, On relativistic wave equations for particles of arbitrary spin in an electromagnetic field, Proc.R.Soc.London, Ser. A, 173 (1939) 211-232; L.P.S. Singh, C.R. Hagen, Lagrangian formulation for arbitrary spin. 1. The bosonic case, Phys. Rev. D9 (1974) 898-909; Lagrangian formulation for arbitrary spin. 2. The fermionic case, Phys. Rev. D9 (1974) 910-920.

[24] E.S. Fradkin, G.A. Vilkovisky, Quantization of relativistic systems with constraints, Phys. Lett. B55 (1975) 224-226; I.A. Batalin, G.A. Vilkovisky, Relativistic S-matrix of dynamical systems with boson and fermion constraints, Phys. Lett. B69 (1977) 309-312; I.A. Batalin, E.S. Fradkin, Operator quantization of relativistic dynamical systems subject to first class constraints, Phys. Lett. B128 (1983) 303.

[25] I.A. Batalin, E.S. Fradkin, Operator quantization method and abelization of dynamical systems subject to first class constraints, Riv. Nuovo Cimento, 9, No 10 (1986) 1; I.A. Batalin, E.S. Fradkin, Operator quantization of dynamical systems subject to constraints. A further study of the construction, Ann. Inst. H. Poincare, A49 (1988) 145; M. Henneaux, C. Teitelboim, Quantization of Gauge Systems, Princeton Univ. Press, 1992.

[26] S. Ouvry, J. Stern, Gauge fields of any spin and symmetry, Phys.Lett. B177 (1986) 335340; A.K.H. Bengtsson, A unified action for higher spin gauge bosons from covariant string theory, Phys.Lett. B182 (1986) 321-325; W. Siegel, B. Zwiebach, Gauge string fields from light cone, Nucl.Phys. B282 (1987) 125; W. Siegel, Gauging Ramond string fields via OSp $(1,1 \mid 2)$, Nucl.Phys. B284 (1987) 632.

[27] A. Pashnev, M. Tsulaia, Description of the higher massless irreducible integer spins in the BRST apperoach, Mod.Phys.Lett. A13 (1998) 1853-1864, [arXiv: hep-th/9803207]; C. Burdik, A. Pashnev, M. Tsulaia, On the mixed symmetry irreducible representations of the Poincare group in the BRST approach, Mod.Phys.Lett. A16 (2001) 731-746, arXiv:hep-th/0101201.

[28] I.L. Buchbinder, A. Pashnev, M Tsulaia, Lagrangian formulation of the massless higher integer spin fields in the AdS background, Phys. Lett. B523 (2001) 338-346, arXiv:hep-th/0109067]; X. Bekaert, I.L. Buchbinder, A. Pashnev, M. Tsulaia, On Higher Spin Theory: Strings, BRST, Dimensional Reductions, Class.Quant.Grav. 21 (2004) 14571464, arXiv:hep-th/0312252.

[29] I.L. Buchbinder, V.A. Krykhtin, A. Pashnev, BRST approach to Lagrangian construction for fermionic massless higher spin fields, Nucl. Phys. B711 (2005) 367-391, arXiv:hep-th/0410215.

[30] I.L. Buchbinder, A. Fotopoulos, A.C. Petkou, M. Tsulaia, Constructing the Cubic Interaction Vertex of Higher Spin Gauge Fields, Phys. Rev. D74 (2006) 105018 arXiv:hep-th/0609082.

[31] I.L. Buchbinder, V.A. Krykhtin, Gauge invariant Lagrangian construction for massive bosonic higher spin fields in D dimensions, Nucl. Phys. B727 (2005) 536-563, arXiv:hep-th/0505092. 
[32] I.L. Buchbinder, V.A. Krykhtin, L.L. Ryskina, H. Takata, Gauge invariant Lagrangian construction for massive higher spin fermionic fields, Phys. Lett. B641 (2006) 386-392, arXiv:hep-th/0603212].

[33] I.L. Buchbinder, V.A. Krykhtin, P.M. Lavrov, Gauge invariant Lagrangian formulation of higher massive bosonic field theory in AdS space, Nucl. Phys. B762 (2007) 344-376, arXiv:hep-th/0608005].

[34] R.R. Metsaev, Gauge invariant formulation of massive totally symmetric fermionic fileds in (A)dS space, Phys. Lett. B643 (2006) 205-212, arXiv:hep-th/0609029.

[35] K. Schoutens, A.Sevrin, P. van Nieuwenhuizen, Quantum BRST Charge for Quadratically Nonlinear Lie Algebras, Commun. Math. Phys. 124 (1989) 87-103; I.L. Buchbinder, P.M. Lavrov, Classical BRST charge for non-linear algebras arXiv:hep-th/0701243.

[36] I.L. Buchbinder, A. Pashnev, M. Tsulaia, Massless higher spin fields in the AdS background and BRST construction for nonlinear algebras, [arXiv; hep-th/0206026]; C. Burdik, O. Navratil, A. Pashnev, On the Fock Space Realizations of Nonlinear Algebras Describing the High Spin Fields in AdS Spaces, [arXiv:hep-th/0206027].

[37] L.D. Faddeev, S.L. Shatoshvili, Realization of the Schwinger term in the Gauss low and the possibility of correct quantization of a theory with anomalies, Phys.Lett. B167 (1986) 225338; I.A. Batalin, E.S. Fradkin, T.E. Fradkina, Another version for operatorial quantization of dynamical systems with iireducible constraints, Nucl. Phys. B314 (1989) 158-174; I.A. Batalin, I.V. Tyutin, Existence theorerm for the effective gauge algebra in the generalized canonical formalism and Abelian conversion of second class constraints, Int.J.Mod.Phys. A6 (1991) 3255-3282; E. Egorian, R. Manvelyan, Quantization of dynamical systems with first and second class constraints, Theor.Math.Phys. 94 (1993) 241-252.

[38] M. Grigoriev, P.H. Damgaard, Superfield BRST charge and the master action, Phys. Lett. B474 (2000) 323-330, arXiv:hep-th/9911092]; G. Barnich, M. Grigoriev, Hamiltonian BRST and Batalin-Vilkovisky formalisms for second quantization of gauge theories, Commun. Math. Phys. 254 (2005) 581-601, arXiv:hep-th/0310083]; D.M. Gitman, P.Yu. Moshin, A.A. Reshetnyak, Local Superfield Lagrangian BRST Quantization, J. Math. Phys. 46 (2005) 072302-01-072302-24, [arXiv:hep-th/0507160]; An Embedding of the BV Quantization into an N=1 Local Superfield Formalism, Phys. Lett. B 621 (2005) 295-308, arXiv:hep-th/0507049].

[39] F.A. Dilkes, M.J. Duff, J.K. Liu, H. Sati, Quantum discontinuity between zero and infinitesimal graviton mass with a Lambda term, Phys.Rev.Lett. 87 (2001) 041301, [arxiv;hep-th/0102093]; M.J. Duff, J.K. Liu, H. Sati, Quantum $M^{2} \rightarrow 2 \Lambda / 3$ discontinuity for massive gravity with a Lambda term, Phys.Lett. B515 (2001) 156 - 160, [arXiv:hepth/0105008]; I.L. Buchbinder, G. de Berredo-Pexoto, I.L. Shapiro, Quantum effects in softly broken gauge theories in curved space-times, Phys. Lett. B649 (2007)454462, arXiv:hep-th/0703189. 